\title{
Molecular Profiling of A375 Human Malignant Melanoma Cells Treated with Kojic Acid and Arbutin
}

\author{
Jung-Feng Hsieh ${ }^{1}$, Shui-Tein Chen ${ }^{2}$ and Sun-Long Cheng ${ }^{3}$ \\ ${ }^{1}$ Department of Food Science, Fu Jen Catholic University \\ 2Institute of Biological Chemistry and Genomics Research Center, Academia Sinica \\ ${ }^{3}$ Department of Plastic Surgery, Chung Shan Medical University, \\ Chung Shan Medical University Hospital \\ Taiwan
}

\section{Introduction}

Malignant melanoma is a serious type of skin cancer that begins in the pigmentation system of the skin. Malignant melanomas, characterized by their high capacity for invasion and metastasis, are one of the most frequent forms of skin cancer. Primary cutaneous melanomas have been divided into four groups based on histopathology: superficial spreading melanoma, nodular melanoma, lentigo maligna melanoma and acral lentiginious melanoma ${ }^{1}$. Superficial spreading melanoma is the most common type of melanoma and grows outwards at first to form an irregular pattern on the skin with an uneven color. It usually starts by spreading out across the surface of the skin in what is known as the radial growth phase. Nodular melanoma occurs most often on the chest or back. It tends to grow deeper into the skin and grows quite deeply if not removed. This type of melanoma is often raised above the rest of the skin surface and feels like a bump. It may be very dark brownblack or black. Lentigo maligna melanoma is most commonly found on the face of elderly people. It grows slowly and may take several years to develop. Acral lentiginious melanoma is usually found on the palms of the hands, soles of the feet or around the toenails. Although melanoma is almost always curable in its early stage, it may be too late if the melanomas spread to other parts of the body. Early diagnosis of melanoma is therefore very important, and it is necessary to develop additional methods of melanoma therapy ${ }^{2}$.

Malignant melanoma usually develops from the transformation and proliferation of melanocytes that normally reside in the basal cell layer of the epidermis. It also develops when the melanocytes no longer respond to normal control mechanisms of cellular growth. The melanocytes may then invade nearby structures or spread to other organs in the body (metastasis), where again they invade and compromise the function of that organ. To better understand the molecular and cellular mechanisms involved in the progression of cutaneous melanoma, human malignant melanoma cells have been widely used as a melanoma skin model for in vitro testing. This is because it is highly reproducible, quantifiable and easily handled in culture. Not only is it a structural cell model that closely parallels the progression of melanoma in vivo, it is also a cost-effective alternative to animal 
and clinical testing. The antiapoptotic mechanisms regulating cell death have been implicated in conferring drug resistance in tumor cells ${ }^{3}$. Therefore, further knowledge on the nature of this resistance and a better understanding of the signal transduction pathways leading to tumor cell death could allow the identification of new target molecules to overcome drug resistance and improve melanoma therapy. In recent years, toxicogenomics has represented the merging of toxicology with genomics and bioinformatics. There have been significant practical challenges in pharmacology to develop both predictive and mechanism-based toxicology in an effort to identify candidate drugs and toxic agents more quickly and economically ${ }^{4}$.

Kojic acid (5-hydroxy-2-hydroxymethyl-1,4-pyrone) is a secondary metabolic product widely used as a food additive for preventing enzymatic browning of raw crabs and shrimps and as a skin lightening or bleaching agent in cosmetic preparations ${ }^{5,6}$. Kojic acid is also used as an important material in antibiotic and pesticide productions and has been shown to act as a competitive and reversible inhibitor of animal and plant polyphenol oxidases, xanthine oxidase, and D- and some L-amino acid oxidases 7,8 . Acute or subchronic toxicity resulting from an oral dose of kojic acid has never been reported, but convulsions may occur if kojic acid is injected. Continuous administration of high doses of kojic acid in mice resulted in thyroid adenomas in both sexes ${ }^{9}$. Moreover, some reports have evaluated the tumorigenic potential of kojic acid, and the genotoxic risk for humans using kojic acid as a skin-lightening agent has also been studied ${ }^{10}$. However, kojic acid at high doses has certain side effects. For example, it affects thyroid function when given at a massive dose or in a long administration period by inhibiting iodine organification in the thyroid, decreasing triiodothyronine (T3) and thyroxine (T4) levels and increasing thyroid-stimulating hormone $(\mathrm{TSH})^{11}$. Recently, kojic acid was found to be a tumor promoter and an enhancer of hepatocarcinogenesis in rats and in mice ${ }^{12}$. However, the topical use of kojic acid as a skinlightening agent results in minimal exposure that poses a negligible or no risk of genotoxicity or toxicity to the consumer.

Arbutin, a natural compound of hydroquinone beta-D-glucopyranoside, is widely used as an ingredient in skin care products ${ }^{13}$. It is effective in the treatment of various cutaneous hyperpigmentations and inhibits melanogenesis in melanoma cells ${ }^{14}$. However, recent findings have raised serious concerns regarding both the safety and side effects of arbutin. Although the mechanisms of some inhibitory effects of arbutin on melanogenesis in melanoma cells have been elucidated, the comprehensive study of its biological effects on the regulation of malignant melanogenesis through the functional effect on carcinogenesis is not clear and rarely reported on a human genomics level. The general risk factors for melanoma include sun exposure, fair skin that burns easily, blistering sunburn, previous melanoma, previous nonmelanoma skin cancer, family history of melanoma, large numbers of moles and abnormal moles. During the progression of melanoma, different proteolytic enzyme systems, including the plasminogen-activator system and matrix metalloproteinases, play an important role in the degradation and remodeling of the extracellular matrix and basement membranes ${ }^{15}$. Tyrosinase is the main enzyme regulating melanogenesis, and it catalyzes three distinct reactions in the melanogenic pathway: hydroxylation of monophenol (L-tyrosine), dehydrogenation of catechol (L-DOPA) and dehydrogenation of dihydroxyindole. By contrast, catalase is the proteolytic enzyme that regulates the removal of $\mathrm{H}_{2} \mathrm{O}_{2}$, which is a potent inhibitor of tyrosinase. Peroxidase also serves to increase eumelanin polymer formation from monomers in the presence of $\mathrm{H}_{2} \mathrm{O}_{2}$ and metal ions, especially copper, which enhance the conversion of monomers to polymers ${ }^{16}$. Thus, changes 
in enzyme levels, including modifications in protein- and gene-expression levels, influence melanogenesis in melanomas. The complex regulatory control of the biosynthesis machinery involved in melanogenesis also includes receptor-mediated pathways activated by hormones, neurotransmitters, cytokines, growth factors and eicosanoids. Additionally, receptor-independent mechanisms are activated or modified by nutrients, micromolecules, microelements, $\mathrm{pH}$, cation and anion concentrations, and the oxidoreductive potential in the physicochemical milieu. Soluble factors can reach target melanocytes by circulation, by release from nerve endings or by local production to act as positive or negative regulators of melanogenesis.

Toxicogenomic applications are increasingly used to evaluate preclinical drug safety and to explain toxicity associated with compounds at the mechanistic level. Recently, a highthroughput DNA microarray technique gained an important role in genomic research by allowing the simultaneous study of the function of thousands of genes showing differential gene expression profiles. This opened the door to the discovery of biomarkers or special gene markers intended for pharmaceutical applications and disease therapy ${ }^{17}$. Highthroughput DNA microarray technology is a powerful tool for genomic research because it allows the study of the function of thousands of genes simultaneously, reveals different gene expression profiles and elucidates the exact mechanisms and defects in genetic aberrations. Recently, microarrays have been used to analyze gene expression profiles of human melanomas. However, only a few such analyses have been reported in A375 melanoma cells. For example, the metastasis of A375 melanoma cells has been studied in nude mice by microarray analysis ${ }^{18}$.

Proteomics is the study of the proteome, the protein complement of the genome. The terms "proteomics" and "proteome" were coined by Marc Wilkins and colleagues in the early 1990s and mirror the terms "genomics" and "genome," which describe the entire collection of genes in an organism ${ }^{19}$. In fact, the proteomic approach is a powerful tool for the simultaneous determination of the protein composition of complex samples. Proteomics consists of several tools, including two-dimensional polyacrylamide gel electrophoresis (2-D PAGE) and mass spectrometry20. 2-D PAGE takes advantage of two unique biochemical characteristics of proteins, combining isoelectric focusing (IEF), which separates proteins according to their isoelectric point, with SDS-PAGE, which further separates them according to their molecular mass. 2-D PAGE allows simultaneous detection and quantification of up to thousands of protein spots on the same gel ${ }^{21}$. Mass spectrometry has been in use for many years, but it could not be applied to macromolecules such as proteins and nucleic acids. The $\mathrm{m} / \mathrm{z}$ measurements are taken on molecules in the gas phase, and the heating or other treatment needed to convert a macromolecule to the gas phase usually caused its rapid decomposition. In 1988, two different techniques were developed to overcome this problem. In one of the techniques, proteins are placed in a light-absorbing matrix. With a short pulse of laser light, the proteins are ionized and then desorbed from the matrix into the vacuum system. This process, known as matrix-assisted laser desorption/ionization mass spectrometry (MALDI MS), has been successfully used to measure the mass of a wide range of macromolecules 22 . MALDI time of flight (TOF) MS is advantageous due to the rapid and direct analysis of complex mixtures and its tolerance to buffer salts or detergents in the samples.

Although the tumorigenic potential and some genotoxic effects of kojic acid and arbutin on human skin cell lines have been widely studied, the effect of these compounds on gene and protein expression levels that may be involved in many biological functions in human skin 
has never been reported. In this study, we used DNA microarrays and 2-D PAGE to investigate the biological effects of kojic acid and arbutin on gene and protein expression profiles of A375 human malignant melanoma cells and on melanocytic tumorigenesis and other related side effects of cancer therapy. These candidate genes and proteins may consequently aid in the development of early diagnostic and therapeutic applications.

\section{Toxicogenomic analysis of kojic acid-treated $A 375$ melanoma cells}

\subsection{Inhibitory effect of kojic acid on A375 melanoma cells}

Previous examination showed that cell growth of A375 melanoma cells was directly inhibited by increasing kojic acid concentrations ${ }^{18}$. After treatment with kojic acid for $72 \mathrm{~h}$, the highest concentration of kojic acid $(1000 \mu \mathrm{g} / \mathrm{ml})$ inhibited A375 melanoma cell growth less than $40 \%$, whereas the lower concentrations of $0.32,1.6,8$ and $40 \mu \mathrm{g} / \mathrm{ml} \mathrm{kojic} \mathrm{acid}$ inhibited A375 melanoma cell growth less than 20\% (Fig. 1).

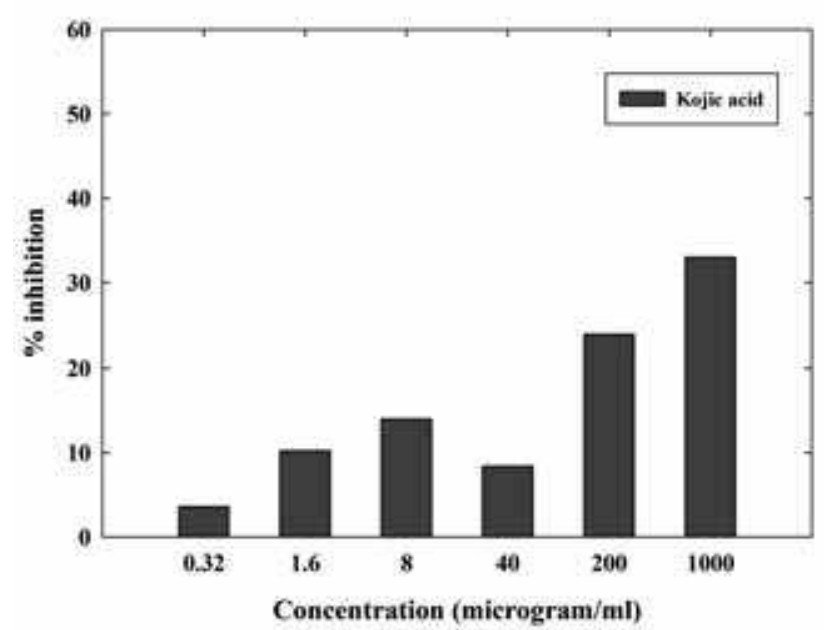

Fig. 1. Effect of different kojic acid concentrations on the growth inhibition of A375 cells at $72 \mathrm{~h}$

These results indicate that the inhibition of A375 melanoma cell growth was not strongly affected by all concentrations of kojic acid. In addition, there was no morphological change of A375 melanoma cells over $24 \mathrm{~h}$ in the presence of $8 \mu \mathrm{g} / \mathrm{ml}$ kojic acid, which is a mild concentration and the recommended dosage of kojic acid for human skin safety 24 . To study the early-stage gene expression profile of A375 melanoma cells following stimulation by kojic acid, comparable to conditions used in human skin therapy, we chose the $24 \mathrm{~h}$ time point for the following microarray analysis.

\subsection{Microarray analysis of kojic acid-treated A375 melanoma cells}

Messenger RNA from control and kojic acid-treated A375 melanoma cells was labeled with Cy3- or Cy5-dCTP, respectively, using a single round of reverse transcription and hybridization with the oligonucleotide microarray chip. The microarray results showed different fluorescence intensities of $\mathrm{Cy} 3$ and $\mathrm{Cy} 5$ depending on the gene expression level ${ }^{18}$. 


\begin{tabular}{|c|c|c|c|}
\hline Gene name & Accession No. & Ratio & Description \\
\hline PSCDBP & NM_004288 & 15.34 & Pleckstrin homology, Sec7 and coiled-coil domains \\
\hline TM4SF3 & NM_004616 & 10.99 & Transmembrane 4 superfamily member 3 (TM4SF3), mRNA \\
\hline KRT7 & NM_005556 & 10.71 & Keratin 7 (KRT7), mRNA \\
\hline KLHL13 & NM_033495 & 10.13 & Kelch-like 13 (Drosophila) (KLHL13), mRNA \\
\hline WBSCR19 & NM_175064 & 9.58 & Williams Beuren syndrome chromosome region 19 (WBSCR19) \\
\hline ВC031966 & ВC031966 & 8.53 & cDNA clone MGC:43036 IMAGE:4839025, complete cds. \\
\hline CRSP2 & NM_004229 & 6.23 & Cofactor required for Spl transcriptional activation, subunit 2 (CRSP2) \\
\hline FGF12 & NM_004113 & 5.95 & Fibroblast growth factor 12 (FGF12), transcript variant 2 , mRNA \\
\hline FLJ46156 & NM_198499 & 5.78 & FLJ46156 protein (FLJ46156), mRNA \\
\hline ENST00000316004 & ENST00000316004 & 5.75 & Olfactory receptor $4 \mathrm{H} 12$ (Fragment). \\
\hline SPP1 & NM_000582 & 5.38 & Secreted phosphoprotein 1 (SPP1), mRNA \\
\hline PLAGL1 & NM_002656 & 5.14 & Pleiomorphic adenoma gene-like 1 (PLAGL1) \\
\hline MGC35118 & NM_152453 & 5.07 & Hypothetical protein MGC35118 (MGC35118), mRNA \\
\hline TTC9 & D86980 & 4.83 & mRNA for KIAA0227 gene, partial cds. \\
\hline $\mathrm{AQP1}$ & NM_000385 & 4.50 & Aquaporin I (channel-forming integral protein, $28 \mathrm{kDa}$ ) (AQPI) \\
\hline PHCA & NM_018367 & 4.47 & Phytoceramidase, alkaline (PHCA), mRNA \\
\hline FBXO16 & NM_172366 & 4.21 & F-box only protein 16 (FBXO16), mRNA \\
\hline PARG & NM_003631 & 4.05 & Poly (ADP-ribose) glycohydrolase (PARG), mRNA \\
\hline SOAT2 & NM_003578 & 4.02 & Sterol O-acyltransferase 2 (SOAT2), mRNA \\
\hline THC1991976 & THC1991976 & 3.67 & AF244540 immunodominant membrane protein precursor \\
\hline PSCD3 & NM_004227 & 3.49 & Pleckstrin homology, Sec7 and coiled-coil domains 3 (PSCD3), mRNA \\
\hline HISTIH2AC & NM_003512 & 3.34 & Histone 1, H2ac (HISTIH2AC), mRNA \\
\hline FLJ 23018 & NM_024810 & 3.32 & Hypothetical protein FLJ23018 (FLJ23018), mRNA \\
\hline PPP4R1 & NM_005134 & 3.01 & Protein phosphatase 4, regulatory subunit 1 (PPP4R1), mRNA \\
\hline CPE & NM_001873 & 2.98 & Carboxypeptidase E (CPE), mRNA \\
\hline C6orf 128 & NM_145316 & 2.97 & Chromosome 6 open reading frame 128 (C6orf128), mRNA \\
\hline CCARI & NM_018237 & 2.91 & Cell division cycle and apoptosis regulator 1 (CCAR1), mRNA \\
\hline FBN2 & NM_001999 & 2.86 & Fibrillin 2 (congenital contractural arachnodactyly) (FBN2), mRNA \\
\hline IL22RAI & NM_021258 & 2.85 & Interleukin 22 receptor, alpha 1 (IL22RA1), mRNA \\
\hline F10 & NM_000504 & 2.80 & Coagulation factor $\mathrm{X}(\mathrm{F} 10)$, mRNA \\
\hline GPR32 & NM_001506 & 2.79 & G protein-coupled receptor 32 (GPR32), mRNA \\
\hline PDCDILG1 & NM_014143 & 2.65 & Programmed cell death 1 ligand 1 (PDCDILG1), mRNA \\
\hline HIP1 & NM_005338 & 2.64 & Huntingtin interacting protein 1 (HIP1), mRNA \\
\hline JMJD2D & NM_018039 & 2.61 & Jumonji domain containing 2D (JMJD2D), mRNA \\
\hline ARMC4 & AK001238 & 2.57 & cDNA FLJ10376 fis, clone NT2RM2001982 \\
\hline CAB39L & NM_030925 & 2.54 & Calcium binding protein 39-like (CAB39L), mRNA \\
\hline FLJ13089 & NM_024953 & 2.54 & Hypothetical protein FLJ13089 (FLJ13089), mRNA \\
\hline OTX1 & NM_014562 & 2.51 & Orthodenticle homolog 1 (Drosophila) (OTX1), mRNA \\
\hline COLQ & NM_080542 & 2.49 & Collagen-like tail subunit of asymmetric acetylcholinesterase \\
\hline PDCL & NM_005388 & 2.44 & Phosducin-like (PDCL), mRNA \\
\hline ENST00000307033 & ENST00000307033 & 2.42 & Olfactory receptor $4 \mathrm{D} 5$. \\
\hline FLJ23311 & NM_024680 & 2.41 & FLJ23311 protein (FLJ23311), mRNA \\
\hline RASSF3 & AK129920 & 2.39 & cDNA FLJ26410 fis, clone HRT09622 \\
\hline LOC220929 & NM_182755 & 2.34 & Hypothetical protein LOC220929 (LOC220929), mRNA \\
\hline EPHA4 & NM_004438 & 2.26 & EphA4 (EPHA4), mRNA \\
\hline HH114 & NM_032499 & 2.23 & Hypothetical protein $\mathrm{HH} 114$ (HH114), mRNA \\
\hline DOCK4 & AB018259 & 2.14 & mRNA for KIAA0716 protein, partial cds. \\
\hline CPNE5 & NM_020939 & 2.11 & Copine V (CPNE5), mRNA \\
\hline
\end{tabular}

Table 1. Up-regulated genes (48 genes, ratio > 2.0) in kojic acid-stimulated A375 cells 
Analysis of gene expression changes at the RNA level using a human 1A oligonucleotide microarray complementary to 20,173 60-mer oligonucleotide probes showed a total number of 83 differentially expressed genes in kojic acid-stimulated A375 melanoma cells, including 48 up-regulated genes (Table 1 , ratio $>2.0$ ) and 35 down-regulated genes (Table 2, ratio < 0.3 ). We suggest that all of the differentially expressed genes may be regulated by various gene networks in the regulation of malignant tumorigenesis.

\subsection{RT-qPCR validation of microarray results}

Genes with a significant change in expression in the array analysis, determined by a mean degree of regulation with $p<0.01$, were chosen for RT-qPCR examination. Differentially expressed genes were grouped by biological function correlated with carcinogenesis and the significantly changed genes were selected according to the order of $\mathrm{P}$ values. The selected genes were validated by RT-qPCR analysis to confirm the microarray data.

RT-qPCR results showed the gene expression levels of seven expressed genes in kojic acidstimulated A375 melanoma cells agreed with the DNA microarray expression data ${ }^{18}$. One gene chosen for RT-qPCR examination was the apolipoprotein B RNA editing deaminase (APOBEC1) gene ${ }^{25}$. The comparison of the gene expression level of sAPOBEC1 by microarray and RT-qPCR is shown in Table 3. This gene was downregulated in kojic acidstimulated A375 melanoma cells and functions as a tumor suppressor in the regulation of carcinogenesis.

\section{Toxicogenomic analysis of arbutin-treated A375 melanoma cells}

\subsection{Inhibitory effect of arbutin on A375 melanoma cells}

A375 melanoma cell growth was directly inhibited by increasing arbutin concentrations 26 . After $72 \mathrm{~h}$, the highest concentration of arbutin $(1000 \mu \mathrm{g} / \mathrm{ml})$ inhibited A375 cell growth up to $40 \%$, while the lower concentrations of $0.32,1.6,8$ and $40 \mu \mathrm{g} / \mathrm{ml}$ arbutin inhibited cell growth by less than $20 \%$ (Fig. 2).

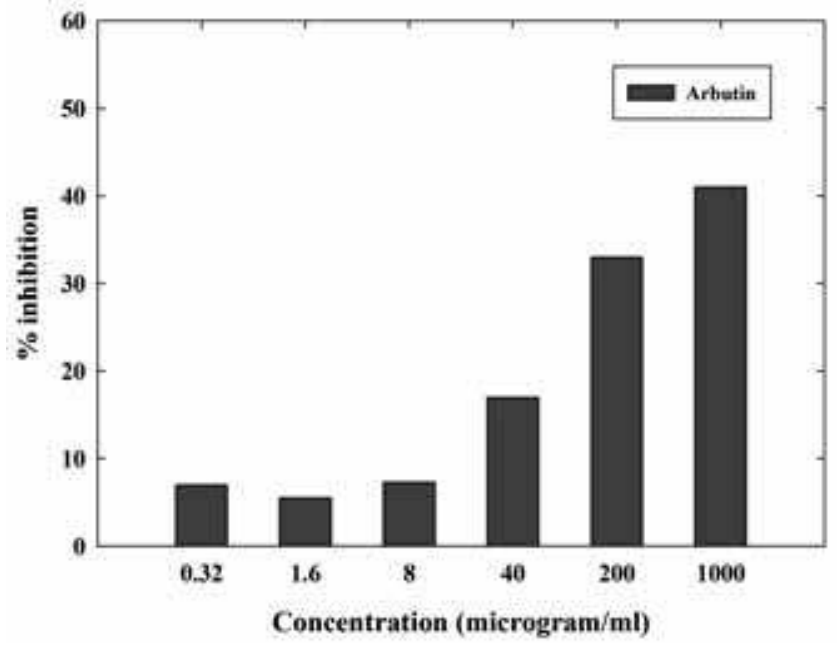

Fig. 2. Effect of arbutin concentrations on the growth inhibition of A375 cells at $72 \mathrm{~h}$ 


\begin{tabular}{|c|c|c|c|}
\hline Gene name & Accession & Ratio & Description \\
\hline DMXL1 & NM_005509 & 0.16 & Dmx-like 1 (DMXL1), mRNA \\
\hline NUDT10 & NM_153183 & 0.16 & Nudix (nucleoside diphosphate linked moiety X)-type motif 10 (NUDT10) \\
\hline SAA4 & NM_006512 & 0.17 & Serum amyloid A4, constitutive (SAA4), mRNA \\
\hline DSPP & NM_014208 & 0.17 & Dentin sialophosphoprotein (DSPP), mRNA \\
\hline IL6ST & NM_002184 & 0.18 & Interleukin 6 signal transducer (gp130, oncostatin M receptor) (IL6ST) \\
\hline PEX5L & NM_016559 & 0.18 & Pex5p-related protein (PEX5R), mRNA \\
\hline BC032472 & BC032472 & 0.18 & Similar to caspase 4 , apoptosis-related cysteine protease \\
\hline AK096677 & AK096677 & 0.19 & cDNA FLJ39358 fis, clone PEBLM2004015 \\
\hline FLJ14297 & NM_024903 & 0.20 & Hypothetical protein FLJ14297 (FLJ14297), mRNA \\
\hline THRB & NM_000461 & 0.20 & Thyroid hormone receptor, beta (THRB), mRNA \\
\hline HLF & NM_002126 & 0.21 & Hepatic leukemia factor (HLF), mRNA \\
\hline APOBEC1 & NM_001644 & 0.22 & Apolipoprotein B mRNA editing enzyme, catalytic polypeptide 1 \\
\hline FGF5 & NM_004464 & 0.22 & Fibroblast growth factor 5 (FGF5), transcript variant 1 , mRNA \\
\hline ACMSD & NM_138326 & 0.22 & Aminocarboxymuconate semialdehyde decarboxylase (ACMSD), mRNA \\
\hline SIN3B & $\mathrm{AB} 014600$ & 0.23 & mRNA for KIAA0700 protein, partial cds. \\
\hline KIAA0565 & $\mathrm{AB} 011137$ & 0.24 & mRNA for KIAA0565 protein, partial cds. \\
\hline MGC34837 & NM_152377 & 0.25 & Hypothetical protein MGC34837 (MGC34837), mRNA \\
\hline TAL1 & NM_003189 & 0.25 & T-cell acute lymphocytic leukemia 1 (TAL1), mRNA \\
\hline SLC17A2 & NM_005835 & 0.25 & Solute carrier family 17 (sodium phosphate), member 2 (SLC17A2) \\
\hline CPB1 & NM_001871 & 0.25 & Carboxypeptidase B1 (tissue) (CPB1), mRNA \\
\hline USP44 & NM_032147 & 0.26 & Ubiquitin specific protease 44 (USP44), mRNA \\
\hline ZNF192 & NM_006298 & 0.26 & Zinc finger protein 192 (ZNF192), mRNA \\
\hline $\mathrm{PAH}$ & NM_000277 & 0.27 & Phenylalanine hydroxylase $(\mathrm{PAH}), \mathrm{mRNA}$ \\
\hline FLJ14503 & NM_152780 & 0.28 & Hypothetical protein FLJ14503 (FLJ14503), mRNA \\
\hline $\mathrm{ZP} 2$ & NM_003460 & 0.29 & Zona pellucida glycoprotein 2 (sperm receptor) (ZP2), mRNA \\
\hline ST18 & NM_014682 & 0.29 & Suppression of tumorigenicity 18 (ST18), mRNA \\
\hline PDZK 10 & $\mathrm{AB} 002314$ & 0.29 & mRNA for KIAA0316 protein, partial cds. \\
\hline TCN1 & NM_001062 & 0.29 & Transcobalamin I (vitamin B12 binding protein, R binder family) (TCN1) \\
\hline $\mathrm{ABCA} 13$ & NM_152701 & 0.29 & ATP binding cassette gene, sub-family $\mathrm{A}(\mathrm{ABCl})$, member 13 ( $\mathrm{ABCA} 13)$ \\
\hline $\mathrm{ABCC} 13$ & NM_138726 & 0.29 & ATP-binding cassette, sub-family C (CFTR/MRP), member 13 (ABCC13) \\
\hline ADAM20 & NM_003814 & 0.29 & A disintegrin and metalloproteinase domain 20 (ADAM20), mRNA \\
\hline AF090929 & AF090929 & 0.29 & Clone HQ0477 PRO0477p mRNA, complete cds. \\
\hline D82326 & D82326 & 0.29 & mRNA for $\mathrm{Na}^{+}$-independent neutral and basic amino acid transporter \\
\hline LILRB5 & NM_006840 & 0.29 & Leukocyte immunoglobulin-like receptor, subfamily B, mRNA \\
\hline MFAP4 & NM_002404 & 0.29 & Microfibrillar-associated protein 4 (MFAP4), mRNA \\
\hline
\end{tabular}

Table 2. Down-regulated genes (35 genes, ratio < 0.3) in kojic acid-stimulated A375 cells

A375 cell growth was not strongly affected by all arbutin concentrations. In addition, there were no morphological changes in cells treated with $0.32-40 \mu \mathrm{g} / \mathrm{ml}$ arbutin. According to the safety recommendations for a $1 \%$ prescription drug in human skin care products, the concentration of $8 \mu \mathrm{g} / \mathrm{ml}$ arbutin is safe and within the recommended concentration ${ }^{27}$. Also, there was no morphological change in the cells within $24 \mathrm{~h}$ in the presence of $8 \mu \mathrm{g} / \mathrm{ml}$ arbutin, and the inhibition of cell growth was less than $10 \%$. However, the biological effect of arbutin on the gene expression profile in A375 melanoma cells and other genotoxic side effects have never been reported. Therefore, a purpose of this study was to investigate the genotoxic effect of arbutin on human skin and on tumorigenesis. 


\begin{tabular}{|c|c|c|c|c|}
\hline Gene name & $P$ value & Microarray & RT-qPCR & (primer sequence) \\
\hline \multirow{2}{*}{ APOBECI } & \multirow{2}{*}{$<0.0001$} & \multirow{2}{*}{0.22} & \multirow{2}{*}{0.12} & $\begin{array}{l}\text { Forward } \\
\text { 5:-FGGATGATGITGIACGCACIGG-3. }\end{array}$ \\
\hline & & & & $\begin{array}{l}\text { Reverse } \\
\text { S:-TGGCGOAATCGTITGOTAATGG.3? }\end{array}$ \\
\hline
\end{tabular}

Table 3. Comparison of gene expression levels of the APOBEC1 gene in kojic acid-treated A375 cells by microarray and RT-qPCR analysis

\subsection{Microarray analysis of arbutin-treated A375 melanoma cells}

The microarray results show different fluorescence intensities of $\mathrm{Cy} 3$ (control) and $\mathrm{Cy} 5$ (arbutin-treated A375 melanoma cells), corresponding to the expression level of thousands of genes. The differences in Cy3 and Cy5 signal intensities with a p-value of less than 0.01 $(p<0.01)$ were considered to be significantly different 26 .

\begin{tabular}{|c|c|c|c|}
\hline Gene name & Accession & Ratio & Description \\
\hline KLHL13 & NM_033495 & 9.31 & Kelch-like 13 (Drosophila) (KLHL13) \\
\hline EPX & NM_000502 & 8.42 & Eosinophil peroxidase (EPX) \\
\hline $\mathrm{TSC} 1$ & NM_000368 & 8.31 & Tuberous sclerosis 1 (TSC1) \\
\hline SCNIB & NM_001037 & 6.32 & Sodium channel, voltage-gated, type I, beta (SCNIB) \\
\hline GAGED3 & NM_130777 & 4.54 & G antigen, family D, 3 (GAGED3) \\
\hline IL3RA & NM_002183 & 4.50 & Interleukin 3 receptor, alpha (low affinity) (IL3RA) \\
\hline FBXO16 & NM_172366 & 4.22 & F-box only protein 16 (FBXO16) \\
\hline VPS39 & NM_015289 & 4.10 & Vacuolar protein sorting 39 (VPS39) \\
\hline PARG & NM_003631 & 4.05 & Poly (ADP-ribose) glycohydrolase (PARG) \\
\hline NRP2 & NM_201266 & 3.94 & Neuropilin 2 (NRP2), transcript variant 1 , mRNA \\
\hline ANKRD23 & NM_144994 & 3.65 & Ankyrin repeat domain 23 (ANKRD23) \\
\hline FLJ13611 & NM_024941 & 3.61 & Hypothetical protein FLJ13611 (FLJ13611) \\
\hline THC1991976 & THC1991976 & 3.55 & AF244540 immunodominant membrane protein precursor \\
\hline AEBP2 & NM_153207 & 3.54 & AE binding protein 2 (AEBP2) \\
\hline ZBTB20 & NM_015642 & 3.36 & Zinc finger protein 288 (ZNF288) \\
\hline $\mathrm{NR} 2 \mathrm{~F} 1$ & NM_005654 & 3.27 & Nuclear receptor subfamily 2 , group F, member 1 (NR2F1) \\
\hline KLRC4 & NM_013431 & 3.24 & Killer cell lectin-like receptor subfamily C, member 4 (KLRC4) \\
\hline CAPNS2 & NM_032330 & 3.17 & Calpain small subunit 2 (CAPNS2) \\
\hline APOM & NM_019101 & 3.12 & Apolipoprotein M (APOM) \\
\hline SLC23A3 & NM_144712 & 2.65 & Solute carrier family 23 , member 3 (SLC23A3) \\
\hline C18orf11 & NM_022751 & 2.62 & Chromosome 18 open reading frame 11 ( $\mathrm{C} 18$ orf11) \\
\hline DDEF1 & NM_018482 & 2.58 & Development and differentiation enhancing factor 1 (DDEF 1 ) \\
\hline HIST1H2AC & NM_003512 & 2.55 & Histone 1, H2ac (HIST1H2AC) \\
\hline HH114 & NM_032499 & 2.49 & Hypothetical protein $\mathrm{HH} 114$ (HH114) \\
\hline CPE & NM_001873 & 2.35 & Carboxypeptidase E (CPE) \\
\hline TARDBP & NM_007375 & 2.28 & TAR DNA binding protein (TARDBP) \\
\hline ADAM7 & NM_003817 & 2.20 & A disintegrin and metalloproteinase domain 7 (ADAM7) \\
\hline KIAA1706 & NM_030636 & 2.16 & KIAA1706 protein (KIAAI706) \\
\hline CCARI & NM_018237 & 2.11 & Cell division cycle and apoptosis regulator 1 (CCAR1) \\
\hline CPNE5 & NM_020939 & 2.10 & Copine V (CPNE5) \\
\hline GPR32 & NM_001506 & 2.04 & G protein-coupled receptor 32 (GPR32) \\
\hline HANDI & NM_004821 & 2.02 & Heart and neural crest derivatives expressed I (HAND1) \\
\hline
\end{tabular}

Table 4. Up-regulated genes (32 genes, ratio > 2.0) in arbutin-stimulated A375 cells 
Analysis of changes in gene expression using the human oligonucleotide array revealed a total of 73 differentially expressed genes, including 32 up-regulated genes (Table 4, ratio > 2.0 ) and 41 down-regulated genes (Table 5 , ratio $<0.3$ ). Therefore, these genes may be useful candidate markers for early diagnostic and therapeutic applications of melanoma carcinogenesis.

\begin{tabular}{|c|c|c|c|}
\hline Gene name & Accession & Ratio & Description \\
\hline$\overline{Z N F 41}$ & NM_153380 & 0.13 & Zinc finger protein 41 (ZNF41) \\
\hline F13B & NM_001994 & 0.16 & Coagulation factor XIII, B polypeptide (F13B) \\
\hline THC1910111 & THC1910111 & 0.17 & BC022679 D13Ertd275e protein \\
\hline HSD3B1 & NM_000862 & 0.17 & Hydroxy-delta-5-steroid dehydrogenase, 3 beta-and steroid delta-isomerase \\
\hline PF4 & NM_002619 & 0.18 & Platelet factor 4 (chemokine (C-X-C motif) ligand 4) (PF4) \\
\hline IL6ST & NM_002184 & 0.18 & Interleukin 6 signal transducer (IL6ST) \\
\hline LOC162967 & NM_207333 & 0.19 & Hypothetical protein LOC162967 (LOC162967) \\
\hline RFPL3 & NM_006604 & 0.20 & Ret finger protein-like 3 (RFPL3) \\
\hline NTF5 & NM_006179 & 0.20 & Neurotrophin 5 (neurotrophin 4/5) (NTF5) \\
\hline GPR155 & NM_152529 & 0.20 & G protein-coupled receptor 155 (GPR155) \\
\hline CASP8 & NM_033357 & 0.20 & Caspase 8, apoptosis-related cysteine protease (CASP8) \\
\hline C20orf17 & NM_173485 & 0.20 & Chromosome 20 open reading frame 17 (C20orf17) \\
\hline AK025116 & AK025116 & 0.20 & cDNA: FLJ21463 fis, clone COL04765 \\
\hline MYO3B & NM_138995 & 0.21 & Myosin IIIB (MYO3B) \\
\hline ZNF167 & NM_018651 & 0.22 & Zinc finger protein 167 (ZNF167) \\
\hline RFPL2 & NM_006605 & 0.22 & Ret finger protein-like 2 (RFPL2) \\
\hline PDZK1 & NM_002614 & 0.22 & PDZ domain containing 1 (PDZK1) \\
\hline NEGRI & NM_173808 & 0.22 & Neuronal growth regulator 1 (NEGR1) \\
\hline GPC3 & NM_004484 & 0.22 & Glypican 3 (GPC3) \\
\hline DMXL1 & NM_005509 & 0.22 & Dmx-like 1 (DMXL1) \\
\hline ZNF141 & NM_003441 & 0.23 & Zine finger protein 141 (clone pHZ-44) (ZNF141) \\
\hline SLC7A3 & NM_032803 & 0.23 & Solute carrier family 7 , member 3 (SLC7A3) \\
\hline SEMG1 & NM_003007 & 0.23 & Semenogelin I (SEMGI) \\
\hline L17325 & L17325 & 0.23 & Pre-T/NK cell associated protein (1D12A2) \\
\hline FMNL3 & NM_175736 & 0.23 & Formin-like 3 (FMNL3) \\
\hline CPBI & NM_001871 & 0.23 & Carboxypeptidase B1 (tissue) (CPB1) \\
\hline ADAMTS9 & NM_020249 & 0.23 & A disintegrin-like and metalloprotease with thrombospondin type 1 motif \\
\hline FGF5 & NM_004464 & 0.24 & Fibroblast growth factor 5 (FGF5) \\
\hline C14orf148 & NM_138791 & 0.24 & Hypothetical protein FLJ32809 (LOC122945) \\
\hline BMPR1B & NM_001203 & 0.24 & Bone morphogenetic protein receptor, type IB (BMPR1B) \\
\hline $\mathrm{ABCA} 10$ & NM_080282 & 0.24 & ATP-binding cassette, sub-family $\mathrm{A}(\mathrm{ABC} 1)$, member 10 ( $\mathrm{ABCA} 10)$ \\
\hline PKHD1 & NM_138694 & 0.25 & Polycystic kidney and hepatic disease 1 (PKHD1) \\
\hline NM_152768 & NM_152768 & 0.25 & Hypothetical protein FLJ25378 (FLJ25378) \\
\hline $\mathrm{ABCC} 13$ & NM_138726 & 0.25 & ATP-binding cassette, sub-family C (CFTR/MRP), member 13 (ABCC13) \\
\hline SLC15A3 & NM_016582 & 0.27 & Solute carrier family 15 , member 3 (SLC15A3) \\
\hline LGALS14 & NM_020129 & 0.27 & Placental protein 13-like protein (PPL13) \\
\hline C6orf97 & NM_025059 & 0.27 & Chromosome 6 open reading frame 97 (C6orf97) \\
\hline AX721299 & AX721299 & 0.27 & Sequence 259 from Patent WO0220754 \\
\hline C15orf26 & NM_173528 & 0.28 & Hypothetical protein FLJ38615 (FLJ38615) \\
\hline ADAM20 & NM_003814 & 0.29 & A disintegrin and metalloproteinase domain 20 (ADAM20) \\
\hline ACMSD & NM_138326 & 0.29 & Aminocarboxymuconate semialdehyde decarboxylase (ACMSD) \\
\hline
\end{tabular}

Table 5. Down-regulated genes (41 genes, ratio < 0.3) in arbutin-stimulated A375 cells 


\section{Proteomic analysis of kojic acid-treated A375 melanoma cells}

\subsection{2-D PAGE of A375 melanoma cells following kojic acid treatment}

To investigate the anticancer effect of kojic acid on the protein expression pattern of A375 melanoma cells, cells were treated with kojic acid for 24, 48 and $72 \mathrm{~h}$ and analyzed by 2-D PAGE28. The 2-D gel images of the untreated control and kojic acid-treated A375 melanoma cells are shown in Fig. 3.

Most of the proteins have $\mathrm{pI}$ ranges of 3.5-5.0 or 6.5-8.5 with molecular weights of more than $25 \mathrm{kDa}$. Using PDQuest image analysis software, approximately 540 spots were detected in control and treatment groups with no significant difference of the mean numbers among groups. Most of the differentially expressed protein spots have a high molecular weight of 45-100 kDa, especially at acidic $\mathrm{pH}$.
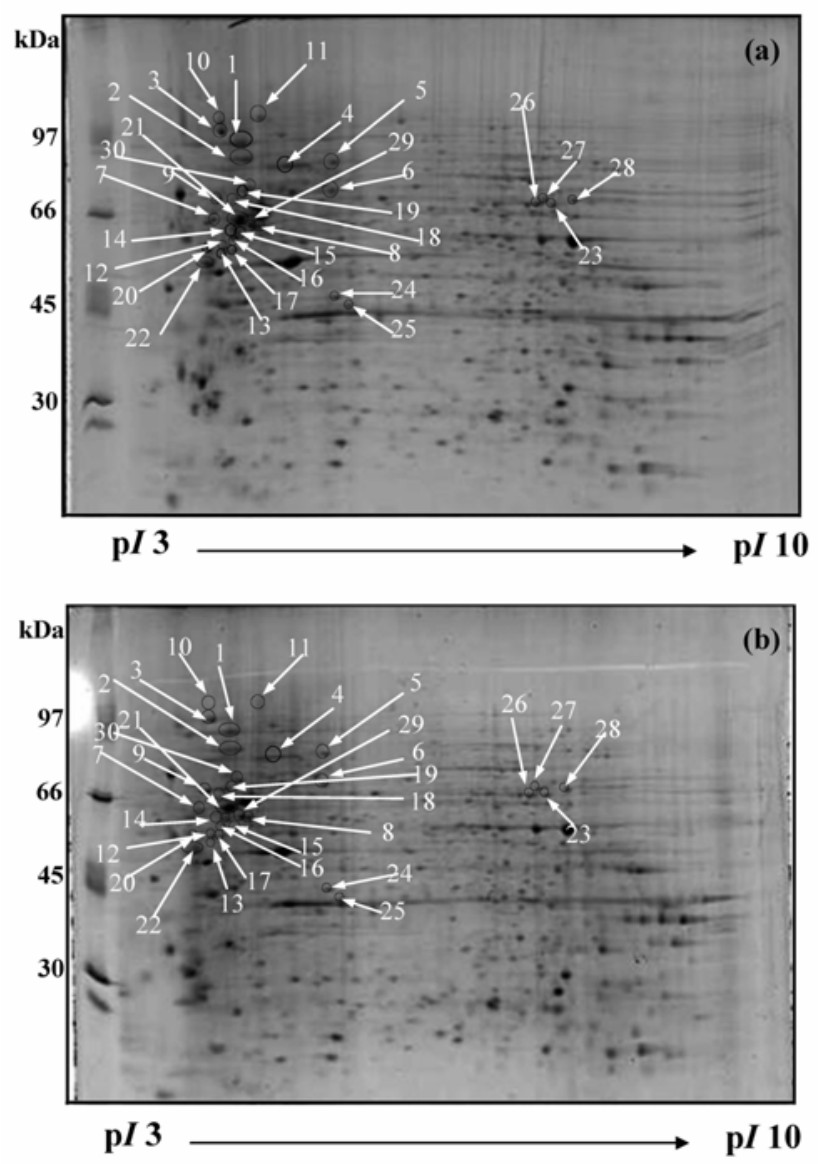

Fig. 3. 2-D PAGE images of A375 human malignant melanoma cells after control (a) and $72 \mathrm{~h}$ treatment (b) with arbutin. The images in the panels are the regions showing differentially expressed spots for protein identification. The pointed arrows indicate the protein spots identified in this study 
To study the biological effect of kojic acid on late-stage protein expression in A375 melanoma cells, the time point of $72 \mathrm{~h}$ was chosen to quantify protein expression changes between the control and treatment groups, and the differentially expressed protein spots were used to further identify the proteins. There were 30 differentially expressed protein spots, including 2 up-regulated protein spots, for which the threshold of change in expression level was 1.5-fold, and 28 down-regulated protein spots, for which the threshold of change in expression level was 0.9 -fold. Seven interesting proteins that have biological functions associated with the p53 tumor suppressor protein or are involved in tumor biology were then selected for validation by Western blot or RT-PCR.

\subsection{Protein identification of differentially expressed proteins}

With the use of MASCOT protein identification search software for identifying both peptide mass fingerprinting (PMF) and MS/MS ion mass data, 30 differentially expressed protein spots were successfully identified ${ }^{28}$. A list of identified proteins, the apparent and theoretical MW, $\mathrm{pI}$ and fold change in expression level (up- or down-regulation) are shown in Table 6. Most of identified proteins were downregulated in kojic acid-treated A375 melanoma cells when compared to the control group. There were multiple isoforms of 5 heat shock proteins, 2 endoplasmin precursors, 2 protein disulfide isomerases, 6 vimentins and 2 aldehyde dehydrogenases, which comprised about $56.7 \%$ of the identified proteins, which might be due to post-translational modifications. In addition, the prediction of protein interaction partners is shown in Table 6. The identified proteins were found at different locations within the cell. The proteins have diverse functions, especially in protein binding, and play key roles in virtually all biological processes, including apoptosis, mammalian cell-cycle progression, regulation of angiogenesis, regulation of cell proliferation, regulation of cell differentiation and the immune response. Interestingly, 11 identified proteins ( $36.7 \%$ of total proteins), including heat shock proteins (HS90B, GRP75, GRP78, ENPL (x2), HSP7C), Tcomplex protein 1 subunit epsilon (TCPE), protein disulfide isomerases (PDIA6, PDIA1), nucleolin (NUCL) and annexin A11 (ANX11), are chaperone proteins, which were found in melanosomes from stage I to stage IV with different functions.

\subsection{Validation by Western blotting and RT-PCR}

Of the identified proteins, GRP75, heat shock protein HS90B, ENPL and pyruvate kinase isozyme M1/M2 (KPYM) were validated by RT-PCR, while GRP75, VIME and serine/threonine-protein phosphatase 2A (2AAA) were validated by Western blotting (Fig. 4).

Protein and gene expression levels of 7 differentially expressed proteins were in agreement with the 2-D PAGE expression data ${ }^{28}$. The proteins GRP75, VIME, HS90B and ENPL were downregulated in kojic acid-treated A375 melanoma cells, while the proteins 2AAA and KPYM were upregulated.

\section{Proteomic analysis of arbutin-treated $A 375$ melanoma cells}

\subsection{2-D PAGE of A375 melanoma cells following arbutin treatment}

The 2-D PAGE images of A375 melanoma cells without treatment (control) and with arbutin treatment at 24, 48 and $72 \mathrm{~h}$ are shown in Fig. 5. 


\begin{tabular}{|c|c|c|c|c|c|c|c|c|}
\hline $\begin{array}{l}\text { Sopt } \\
\text { No. }\end{array}$ & Protein Name & $\begin{array}{l}\text { Accession } \\
\text { no. }\end{array}$ & $\begin{array}{c}\text { Mr } \\
(\mathbf{E x p})\end{array}$ & $\begin{array}{c}\mathrm{p} I \\
(\mathbf{E x p})\end{array}$ & $\begin{array}{l}\text { Mr } \\
\text { (Cal) }\end{array}$ & $\begin{array}{c}\mathbf{p} I \\
(\mathbf{C a l})\end{array}$ & $\begin{array}{c}\text { Fold- } \\
\text { change }\end{array}$ & Predicted protein interaction partners \\
\hline 1 & Heat shock protein HSP 90-beta & P08238 & 97.00 & 4.20 & 83.2 & 4.97 & -2.50 & $\cdot$ TP53/p53 \\
\hline 2 & Heat shock $70 \mathrm{kDa}$ protein & P11021 & 91.80 & 4.20 & 72.3 & 5.07 & -2.50 & $\cdot$ ENPL $•$ PDIA6 $•$ GRP75 •p53 \\
\hline 3 & Endoplasmin precursor (ENPL) & P14625 & 100.00 & 4.00 & 92.4 & 4.76 & -1.51 & $\begin{array}{l}\cdot \text { GRP75 •GRP78 •PDIA4 receptor } \\
\cdot \text {-Tyrosinase-protein kinase erbB-2 }\end{array}$ \\
\hline 4 & Heat shock cognate $71 \mathrm{kDa}$ (HSP7C) & P11142 & 86.64 & 4.67 & 70.85 & 5.37 & -2.00 & $\begin{array}{l}\text { - Bcl-2 binding athanogene-1, }-3 \\
\text {-HSP86 } \cdot \text { HSP60 }\end{array}$ \\
\hline 5 & $\begin{array}{l}\text { Stress-70 protein, mitochondrial } \\
\text { precursor (GRP75) }\end{array}$ & P38646 & 89.22 & 5.10 & 73.64 & 5.87 & -2.21 & $\begin{array}{l}\cdot \mathrm{p} 53 \cdot \text { DnaJ homologue subfamily A } \\
\text { and B members } \cdot \text { HSP } 90 \mathrm{~B} 1\end{array}$ \\
\hline 6 & $\begin{array}{l}\text { T-complex protein } 1 \text { subunit epsilon } \\
\text { (TCPE) }\end{array}$ & P48643 & 76.32 & 5.10 & 59.63 & 5.45 & -2.20 & $\begin{array}{l}\text {-T-complex protein } 1 \text { different } \\
\text { subunits }\end{array}$ \\
\hline 7 & Endoplasmin precursor (ENPL) & P14625 & 68.00 & 3.95 & 92.41 & 4.76 & -1.10 & $\begin{array}{l}\cdot \text { GRP75 •GRP78 •PDIA4 receptor } \\
\cdot \text { Tyrosinase-protein kinase erbB-2 }\end{array}$ \\
\hline 8 & $\begin{array}{l}\text { Protein disulfide-isomerase A6 } \\
\text { precursor (PDIA6) }\end{array}$ & Q15084 & 63.00 & 4.60 & 48.09 & 4.95 & -2.00 & •Beta-Actin •ERp31 • GRP78 \\
\hline 9 & $\begin{array}{l}\text { Protein disulfide-isomerase precursor } \\
\text { (PDIA1) }\end{array}$ & P07237 & 71.20 & 4.00 & 57.08 & 4.76 & -2.10 & •ERp31 •GRP78 \\
\hline 10 & Nucleolin (NUCL) & P19338 & 102.00 & 4.00 & 76.57 & 4.60 & -2.20 & $\begin{array}{l}\text {-DNA topoisomerase } \cdot \text { p } 53 \\
\text { •Proliferation-associated protein } 2 \mathrm{G} 4\end{array}$ \\
\hline 11 & Vimentin (VIME) & P08670 & 103.00 & 4.40 & 53.62 & 5.06 & -2.25 & $\begin{array}{l}\cdot \mathrm{RAF}-1 \cdot \text { Proteosome subunit alpha } \\
\text { type } 1 \cdot \text { Protein kinase N1 }\end{array}$ \\
\hline 12 & Vimentin (VIME) & P08670 & 58.50 & 4.25 & 53.62 & 5.06 & -2.15 & $\begin{array}{l}\cdot \mathrm{RAF}-1 \cdot \text { Proteosome subunit alpha } \\
\text { type1 } \cdot \text { Protein kinase N1 }\end{array}$ \\
\hline 13 & Vimentin (VIME) & P08670 & 57.00 & 4.22 & 53.62 & 5.06 & -2.10 & $\begin{array}{l}\cdot \mathrm{RAF}-1 \cdot \text { Proteosome subunit alpha } \\
\text { type1 } \cdot \text { Protein kinase N1 }\end{array}$ \\
\hline 14 & Vimentin (VIME) & P08670 & 63.00 & 4.34 & 53.62 & 5.06 & -2.10 & $\begin{array}{l}\cdot \mathrm{RAF}-1 \cdot \text { Proteosome subunit alpha } \\
\text { type } 1 \cdot \text { Protein kinase N1 }\end{array}$ \\
\hline 15 & Vimentin (VIME) & P08670 & 62.50 & 4.40 & 53.62 & 5.06 & -2.00 & $\begin{array}{l}\cdot \mathrm{RAF}-1 \cdot \text { Proteosome subunit alpha } \\
\text { type1 } \cdot \text { protein kinase N1 }\end{array}$ \\
\hline 16 & Vimentin (VIME) & P08670 & 61.30 & 4.30 & 53.62 & 5.06 & -2.15 & $\begin{array}{l}\cdot \text { RAF-1 } \cdot \text { Proteosome subunit alpha } \\
\text { type1 } \cdot \text { Protein kinase N1 }\end{array}$ \\
\hline 17 & Gamma-enolase (EC 4.2.1. 11) & P09104 & 58.00 & 4.17 & 47.24 & 4.91 & -2.00 & $\begin{array}{l}\text {-Pyruvate kinase isoenzyme M1/M2 } \\
\text {-Enzymes in glycolytic pathway }\end{array}$ \\
\hline 18 & $\begin{array}{l}\text { Pyruvate kinase isozymes M1/M2 } \\
\text { (KPYM) }\end{array}$ & P14618 & 71.16 & 4.40 & 57.90 & 7.96 & +2.15 & - Alpha enolase $\cdot$ Beta enolase \\
\hline 19 & $\begin{array}{l}\text { Serine/threonine-protein phosphatase } \\
2 \mathrm{~A} 65 \mathrm{kDa} \text { regulatory subunit A alpha } \\
\text { isoform (2AAA, PP2A) }\end{array}$ & P30153 & 76.30 & 4.25 & 65.18 & 4.96 & +1.52 & $\begin{array}{l}\cdot \text { RAF proto-oncogene serine/threonine } \\
\text { kinase } \cdot \text { Serine/threonine protein } \\
\text { phosphatase isoforms } \\
\cdot \text { Protein KIAA0889 }\end{array}$ \\
\hline 20 & Ribonuclease inhibitor (RINI) & P13489 & 58.50 & 3.90 & 49.94 & 4.71 & -2.18 & $\begin{array}{l}\cdot \mathrm{TNF}-\alpha \cdot \mathrm{TNF}-\mathrm{R} 1 \\
\cdot \text {-NF-kappa-B-essential modulator } \\
\cdot \text {-Kinesin-like protein (KIF1A) }\end{array}$ \\
\hline 21 & Tubulin beta chain (TBB5) & P07437 & 65.00 & 4.40 & 49.64 & 4.78 & -0.99 & $\begin{array}{l}\text {-Mitogen-activated protein kinase } 10 \\
\text { - Dual specificity protein kinase TTK }\end{array}$ \\
\hline 22 & $\begin{array}{l}\text { Melanoma-associated antigen } 4 \\
\text { (MAGA-4) }\end{array}$ & P43358 & 55.00 & 3.90 & 34.91 & 4.68 & -2.30 & $\begin{array}{l}\cdot 26 \mathrm{~S} \text { proteosome regulatory subunit } \\
\text { p } 28 \cdot \mathrm{B} \text { melanoma antigen } 3 \\
\text { precursor } \cdot \text { Cancer/testis antigen } 2 \\
\text { - Programmed cell death protein } 6\end{array}$ \\
\hline 23 & $\begin{array}{l}\text { Annexin A11 (Calcyclin-associated } \\
\text { annexin 50) (ANX11) }\end{array}$ & P50995 & 71.20 & 6.18 & 54.36 & 7.53 & -1.55 & $\begin{array}{l}\text {-40S ribosomal protein } 24 \\
\text {-Calcyclin } \cdot \text { DNA-directed RNA } \\
\text { polymerase III }\end{array}$ \\
\hline 24 & $\begin{array}{l}\text { Eukaryotic translation initiation factor } \\
3 \text { subunit } 2\end{array}$ & Q13347 & 48.00 & 5.10 & 36.48 & 5.38 & -1.00 & $\begin{array}{l}\text {-Eukaryotic translation initiation } \\
\text { factor subunits }\end{array}$ \\
\hline 25 & $\begin{array}{l}\text { 60S acidic ribosomal protein } \mathrm{P} 0 \\
\text { (RLAO) }\end{array}$ & P05388 & 45.50 & 5.15 & 34.25 & 5.71 & -0.95 & $\begin{array}{l}\text { - Ribosomal proteins } \\
\text {-Elongation factor 1-gamma }\end{array}$ \\
\hline 26 & $\begin{array}{l}\text { Inosine-5' monophosphate } \\
\text { dehydrogenase } 2 \text { (EC 1.1.1.205) } \\
\text { (IMDH2) }\end{array}$ & P12268 & 71.16 & 6.00 & 55.77 & 6.44 & -2.00 & $\begin{array}{l}\text {-Inosine-5'monophosphate } \\
\text { dehydrogenase } 1 \cdot \text { GMP synthase } \\
\text {-GMP reductase } 1 \text { and } 2 \\
\text {-AMP deaminase } 1 \text { and } 3\end{array}$ \\
\hline 27 & $\begin{array}{l}\text { Aldehyde dehydrogenase } 1 \mathrm{~A} 3 \text { (EC } \\
1.2 .1 .5)(\mathrm{AL} 1 \mathrm{~A} 3 \text { ) }\end{array}$ & P47895 & 72.00 & 6.25 & 56.07 & 6.99 & -1.02 & $\begin{array}{l}\text {-3-Hydroxyisobutyrate dehydrogenase } \\
\text {-Acetyl-coenzyme A synthetase 2-like }\end{array}$ \\
\hline 28 & $\begin{array}{l}\text { Aldehyde dehydrogenase } 1 \mathrm{~A} 3 \text { (EC } \\
1.2 .1 .5) \text { (AL1A3) }\end{array}$ & P47895 & 72.00 & 6.35 & 56.07 & 6.99 & -0.99 & $\begin{array}{l}\text {-3-Hydroxyisobutyrate dehydrogenase } \\
\text {-Acetyl-coenzyme A synthetase 2-like }\end{array}$ \\
\hline 29 & $\begin{array}{l}\text { ATP synthase subunit beta, } \\
\text { mitochondrial precursor (EC 3.6.3.14) }\end{array}$ & P06576 & 64.00 & 4.50 & 56.52 & 5.26 & -1.00 & $\begin{array}{l}\text {-ATP synthase isoforms } \\
\text {-NADH-ubiquinone oxidoreductase }\end{array}$ \\
\hline 30 & $\begin{array}{l}\text { Rab GDP dissociation inhibitor alpha } \\
\text { (Rab GDI alpha) }\end{array}$ & P31150 & 76.32 & 4.30 & 50.55 & 5.00 & -0.97 & $\begin{array}{l}\cdot \mathrm{RAB} 4 \mathrm{~A} \cdot \mathrm{RAB} 5 \mathrm{~A} \cdot \mathrm{RAB} 2 \\
\cdot \mathrm{RAB} 11 \mathrm{~A} \cdot \mathrm{RAB} 9 \mathrm{~A} \\
\end{array}$ \\
\hline
\end{tabular}

Table 6. Protein identification of differentially expressed proteins in kojic acid-treated A375 cells and the prediction of protein interaction partners 


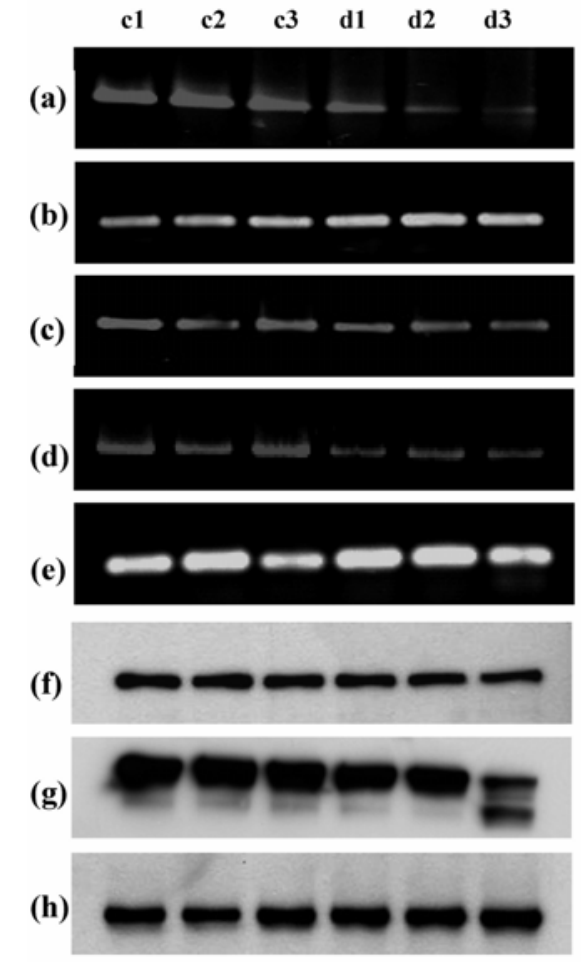

Fig. 4. Validation of differentially expressed proteins by Western blot and RT-PCR analyses. RT-PCR of (a) GRP75, (b) 2AAA, (c) HS90B (d) ENPL and (e) KPYM. Western blot of (f) GRP75, (g) VIME, and (h) 2AAA. c1, c2 and c3 indicate the control groups at 24, 48 and $72 \mathrm{~h}$, respectively. $\mathrm{d} 1, \mathrm{~d} 2$ and $\mathrm{d} 3$ indicate the treatment groups at 24,48 and $72 \mathrm{~h}$, respectively

The molecular weights of the proteins were distributed within the range of 25 to $97 \mathrm{kDa}$, and the differentially expressed protein spots had isoelectric points distributed between acidic and basic $\mathrm{pH}^{29}$. Using PDQuest image analysis software, approximately 540 spots were found in the control and treatment groups with no significant difference between the groups. There were 40 differentially expressed protein spots, including 10 up-regulated protein spots with a threshold of change in expression level of 1.0-fold, and 40 downregulated protein spots with a threshold of change in expression level of 0.9 -fold.

\subsection{Identification of differentially expressed proteins}

Using the MASCOT protein identification search software for identifying both PMF and MS/MS ion mass data, 26 differentially expressed proteins were successfully identified 29. Among these proteins, there were five up-regulated and 15 down-regulated proteins (including their isoforms). Most of the identified proteins were downregulated in arbutintreated A375 melanoma cells, and 13 isoforms of six identical proteins were observed. There were two vimentins, two heterogeneous nuclear ribonucleoproteins A2/B1, two heterogeneous nuclear ribonucleoproteins A1, two peroxiredoxin-1 proteins, three glyceraldehyde-3-phosphate dehydrogenases and two alpha-enolases, accounting for about 

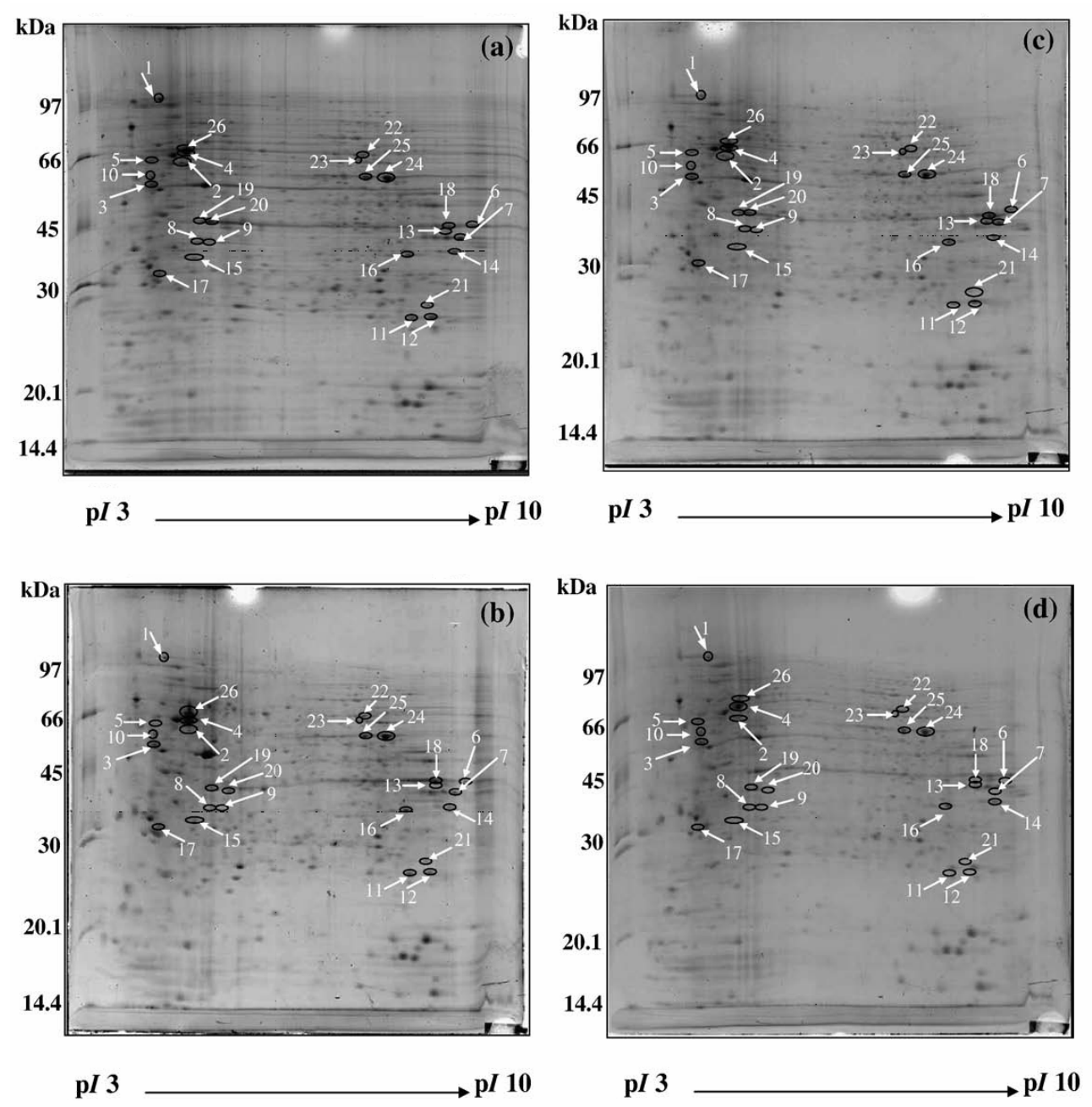

Fig. 5. 2-D PAGE images from A375 human malignant melanoma cells under the following conditions: (a) control, (b) 24-h arbutin treatment, (c) 48-h arbutin treatment and (d) 72-h arbutin treatment. The samples were separated on a homogeneous 12.5\% SDS-PAGE gel using a pH 3-10 NL IPG strip. The pointed arrows indicate the protein spots identified in this study

$50 \%$ of all identified proteins possibly due to post-translational modifications. The protein interaction partners are also predicted. The identified proteins are distributed to many locations within the cell, have diverse functions and play important roles in many biological processes, especially in cancer biology, such as apoptosis, angiogenesis, cell proliferation, cell differentiation and the immune response. Interestingly, five identified proteins, heat shock protein $90 \mathrm{kDa}$ beta member 1 (ENPL), voltage-dependent anion-selective channel protein 1 (VDAC-1), chloride intracellular channel protein 1 (CLIC1), guanine nucleotide- 
binding protein subunit beta (GBLP) and 14-3-3 protein gamma (14-3-3G) (19\% of total proteins), were found to play important roles in apoptosis and signal transduction. Additionally, six identified proteins (nine spots including their isoforms, $34.6 \%$ of total proteins), nuclear ribonucleoprotein $\mathrm{H}$ (HNRH1), ribonucleoproteins A2/B1 (ROA2), ribonucleoprotein A1 (ROA1), ribonuclease inhibitor (RINI), 14-3-3G and alpha-enolase (ENOA), function in nucleic acid processing or transcriptional regulation. Moreover, four identified proteins (seven spots including their isoforms, $27 \%$ of total proteins), malate dehydrogenase (MDHM), glyceraldehyde-3-phosphate dehydrogenase (G3P), glucose-6phosphate 1-dehydrogenase (G6PD) and ENOA are involved in carbohydrate metabolism pathways and have additional functions in the regulation of cell growth and maintenance of cellular functions.

\subsection{Validation by Western blot or RT-PCR}

Of the identified proteins, vimentin (VIME), 14-3-3G, peroxiredoxin-1 (PRDX1), ENPL, inosine-5'-monophosphate dehydrogenase 2 (IMDH2), ENOA, VDAC-1 and p53 were validated by RT-PCR (Fig. 6). The protein and gene expression levels of eight differentially expressed proteins were in agreement with the 2-D PAGE expression data ${ }^{29}$. 14-3-3G, VDAC-1 and p53 were upregulated in the arbutin-treated A375 melanoma cells, whereas VIME, PRDX1, ENPL, IMDH2 and ENOA were downregulated in the arbutin-treated A375 melanoma cells.

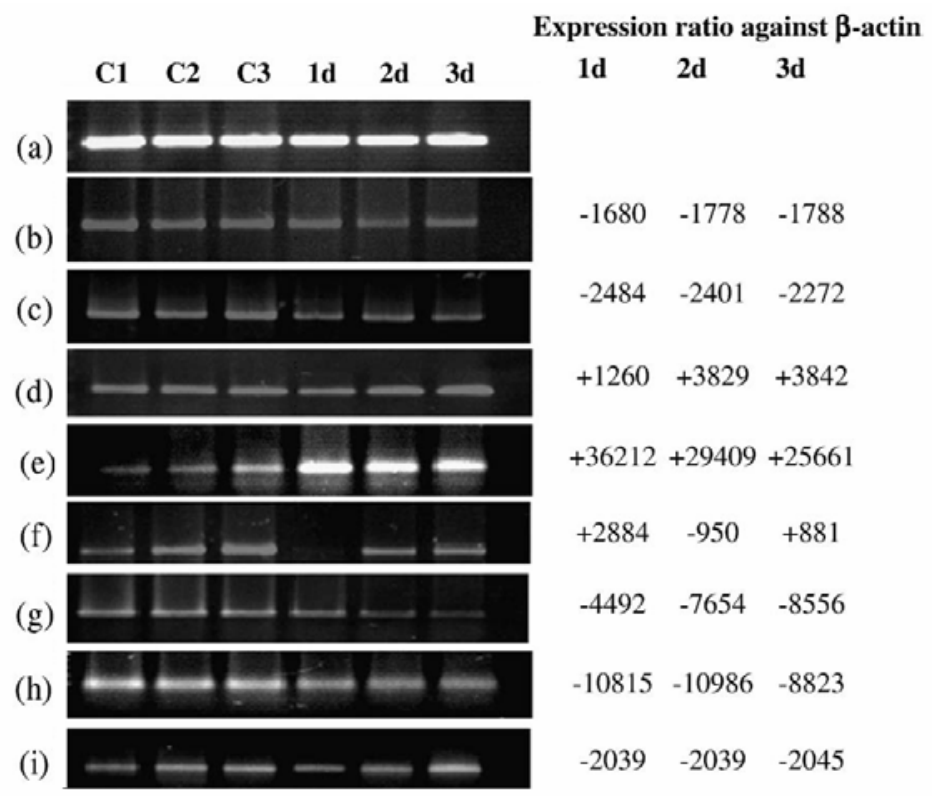

Fig. 6. Validation of differentially expressed proteins by RT-PCR analyses. (a) $\beta$-actin, (b) VIME, (c) ENPL, (d) 14-3-3G, (e) VDAC-1, (f) ENOA, (g) PROX1, (h) IMDH2 and (i) p53. $\mathrm{C} 1, \mathrm{C} 2$ and $\mathrm{C} 3$ indicate the control group at 24,48 and $72 \mathrm{~h}$, respectively. $1 \mathrm{~d}, 2 \mathrm{~d}$ and $3 \mathrm{~d}$ indicate the treatment groups at 24,48 and $72 \mathrm{~h}$, respectively 


\section{Discussion}

Although some of the biological applications and potential effects of kojic acid have been partially elucidated ${ }^{30}$, the effects of kojic acid on a human genomics level, including gene regulatory mechanisms, are not clear and has rarely been reported. In this study, we used A375 human skin malignant melanoma cells to examine the genotoxicity of kojic acid in carcinoma therapy, not including its skin-whitening effect. To examine the genotoxicity effect of kojic acid on the gene expression profile of A375 melanoma cells, microarray technology providing a high-throughput method for easily screening the number of differentially expressed genes was used. It has been used for analysis of gene expression in dermatological studies, including studies of human melanomas ${ }^{31}$. However, the study of gene expression in kojic acid-stimulated A375 melanoma cells by DNA microarray has never been reported. It is challenging to use the high-throughput technology of DNA microarray for studying the effect of kojic acid on the large numbers of differentially expressed genes in A375 melanoma cells and its anti-tumorigenic function to regulate the malignancy of melanoma. We used the human 1A oligonucleotide microarray to analyze gene expression of human skin A375 melanoma cells for the following reasons: (i) the human 1A oligonucleotide microarray is comprised of 20,173 oligonucleotide probes that span conserved exons across transcripts of 18,716 human genes, is compatible with human skin A375 melanoma cells and could screen large numbers of differentially expressed human genes at a single time. (ii) The human $1 \mathrm{~A}$ oligonucleotide microarray uses a convenient two-color labeling procedure that reduces experimental variability by allowing biological samples to be directly compared with each other on the same microarray after undergoing the same hybridization incubation. This microarray platform allowed the analysis of kojic acid-responding genes in A375 melanoma cells that may be used as gene markers of malignant melanoma for diagnostic and therapeutic applications in human skin. We examined the effect of various concentrations $(0.32,1.6,8,40,200$ and $1000 \mu \mathrm{g} / \mathrm{ml})$ of kojic acid on growth inhibition of A375 melanoma cells. Not all kojic acid concentrations strongly inhibited the cell growth of A375 melanoma cells, even at the longer incubation time of $72 \mathrm{~h}$. When using microarray technology to study the overall effects of kojic acid on gene expression of A375 melanoma cells, we used $8 \mu \mathrm{g} / \mathrm{ml}(0.8 \%)$ kojic acid because this is the mildest concentration that could inhibit A375 melanoma cells less than $20 \%$ while avoiding potential differences in gene expression data resulting from the cell death response due to cytotoxicity from higher concentrations of kojic acid. The safe level of kojic acid in human skin care products for general use is up to $1 \%$ without a prescription ${ }^{24}$. Therefore, 8 $\mu \mathrm{g} / \mathrm{ml}$ kojic acid $(0.8 \%)$ is safe for human skin and can be used to study the gene expression profile of A375 melanoma cells and identify the regulatory functions of these genes in carcinogenesis therapy. After microarray data were collected, we used bioinformatic tools to identify the differentially expressed genes and searched Gene Ontology for useful information. The information obtained can be used to further study regulatory mechanisms and gene markers for applications in early cancer diagnosis and therapy. For a number of differentially expressed genes, we selected significant genes involved in carcinogenesis and compared microarray analysis data with RT-qPCR data to validate changes in gene expression. RT-qPCR offered confirmatory quantitative results under stringent conditions. We show the quantification of kojic acid-responding genes from RT-qPCR is in agreement with the DNA microarray expression data. This indicates that the validation of genes in the robust biomarkers lists was done using precise data. These validated genes may become 
useful biomarkers to understand the biochemistry of a drug/small molecule response in future clinical studies. Interestingly, we have found seven significant kojic acid-responsive genes downregulated in kojic acid-stimulated A375 melanoma cells. Most of the genes are related to the regulation of carcinogenesis. Apolipoprotein B mRNA editing enzyme, catalytic polypeptide 1 (APOBEC1), is the central component of an RNA editosome, whose physiological role is the deamination of apoB mRNA cytidine-6666 to uracil in gastrointestinal tissues, thereby creating a premature stop codon ${ }^{32}$. Uncontrolled cytidine deamination could generate inappropriately folded polypeptides, dominant-negative proteins, or mutations in tumor suppressor genes and thus contribute to tumor formation ${ }^{33}$. Overexpression of APOBEC1 has led to the development of a number of different cancers, such as gastrointestinal cancer, colon cancer and heptocellular cancer, in a variety of tissues. This implies that inappropriate expression of a member of the DNA deaminase family could have potential oncogenic activity 34 . Furthermore, most human carcinomas are caused by APOBEC1-mediated mRNA editing 35 . Thus, down-regulated APOBEC1 may potentially deactivate the tumor progression of A375 melanoma cells.

In conclusion, we used high-throughput DNA microarray and RT-qPCR techniques for the global analysis of differentially expressed genes responding to kojic acid in A375 human melanoma cells. The differentially expressed genes were classified depending on cellular localization, molecular function and biological process, and they led to an exploration of the more valuable data on the regulation of melanoma carcinogenesis against kojic acid. In addition, seven of the down-regulated genes that showed significantly different expression in A375 melanoma cells after kojic acid treatment function as tumor suppressors and may disrupt the regulation of melanoma tumorigenesis in human malignant melanoma cells. These genes may be useful markers in further diagnostic and therapeutic applications. Moreover, our findings show the probable side effects and disadvantages of kojic acid due to its effect on the immune system, bone development and maintenance. However, we will further study the effects of kojic acid on biological and molecular mechanisms in human melanoma skin cells, including in other parts of the body, and also examine other biological functions of kojic acid in its cosmetic and/or therapeutic applications. We also examined the effect of different arbutin concentrations on the growth inhibition of A375 melanoma cells and found that the concentrations used did not strongly inhibit cell growth, even though the incubation time was up to $72 \mathrm{~h}$. We used $8 \mu \mathrm{g} / \mathrm{ml}(0.8 \% \mathrm{w} / \mathrm{v})$ arbutin because (i) this was the same concentration of kojic acid used 18 , because this concentration was lower than the safety recommendation of $1 \%$ in prescription human skin care products 36 ; (ii) this concentration inhibited cell growth less than $10 \%$ with no morphological change of cells; and (iii) it avoids changes in gene expression data resulting from the cell death response due to cytotoxicity from a high concentration of arbutin. Therefore, $8 \mu \mathrm{g} / \mathrm{ml}$ arbutin, which is safe for use on human skin, was used to study the genotoxic effect on the gene expression profile of A375 melanoma cells and for examining the differential gene expression and other side effects of altered signaling pathways for cancer therapy. To study the effect of arbutin on the gene expression level of A375 melanoma cells, RNA was isolated from cells $24 \mathrm{~h}$ after arbutin treatment to study gene expression levels with microarray analysis.

We also used high-throughput DNA microarray and bioinformatic tools in a global analysis of differentially expressed genes in arbutin-treated A375 malignant melanoma cells. These genes were classified by Gene Ontology and led to an exploration of more valuable data on the regulation of melanoma carcinogenesis. In addition, four down-regulated genes are candidate tumor suppressor genes in A375 melanoma cells following arbutin treatment. 
They may disrupt the regulation of malignant tumorigenesis in human malignant melanoma cells and may be useful markers for further diagnostic and therapeutic applications. Moreover, the genotoxic effect of arbutin on the gene expression profile of A375 melanoma cells was similar to kojic acid, which indicates a similar regulation of malignant tumorigenesis by these drugs. However, we will conduct further studies on the effects of arbutin on biological and molecular mechanisms in human skin cells. We will also examine other biological characteristics of arbutin for therapeutic applications. According to our study investigating the toxicogenomic effects of kojic acid on the gene expression profile of A375 melanoma cells using microarray analysis, the cell growth inhibition was not strongly affected by all concentrations of kojic acid at a longer treatment time of $72 \mathrm{~h}^{18}$. There was no morphological change of A375 melanoma cells over the $72 \mathrm{~h}$ exposure to 8 $\mu \mathrm{g} / \mathrm{ml}$ kojic acid, which is a mild concentration and the recommended safe dose of kojic acid for human skin. The early stage of the kojic acid stimulatory effect on the gene expression profile of A375 melanoma cells also uncovered some tumor suppressor genes. Although one gene makes one protein, protein modifications leading to changes in biological and physiological functions may not be caused by gene modifications. To examine the effects of kojic acid on protein expression levels in A375 melanoma cells, the same concentration of kojic acid $(8 \mu \mathrm{g} / \mathrm{ml})$ as in the microarray study, which is compliant with the safety recommendation in human skin care products, and various treatment times $(24,48$ and $72 \mathrm{~h}$ ) were used in this study. 2-D PAGE identified 30 differentially expressed proteins that showed a significant change in expression levels following $72 \mathrm{~h}$ of drug treatment. We used combined databases to propose a protein interaction network in which 16 differentially expressed proteins are involved in the regulation of apoptosis via major signaling proteins, such as p53, Ras, MEK/ERK, RAF-1 and Bcl-2. Interestingly, 11 chaperone proteins were downregulated in kojic acid-treated A375 melanoma cells and were found to interact with each other. It is well known that heat shock proteins are required for cell survival during stress and also have key functions in controlling cellular metabolism ${ }^{37}$. In cancer biology, heat shock proteins are expressed at high levels during facilitated tumor cell growth and survival $^{38}$. In addition, GRP75, GRP78 and HSP90 interact with the p53 tumor suppressor ${ }^{39}$. HSP90B has a principal role in the regulation of mitogenesis and cell cycle progression and could interact with HSP70 or GRP75 to participate in the protein degradation process and protection from programmed cell death. HSP7C interacts with the Bcl-2 binding proteins athanogene- 1 and athanogene- 3 and other chaperone proteins, including HS90B and ENPL.

According to our results of down-regulated heat shock proteins in kojic acid-treated A375 melanoma cells, cellular stress in malignant melanoma cells was observed following kojic acid treatment. Kojic acid could suppress the expression of heat shock proteins, which support tumor growth and may lead to an antiapoptosis effect. Alternatively, because a primary mechanism for the regulation of heat shock proteins in normal cells involves the tumor repressor p53 and the related protein p63, the downregulation of heat shock proteins may be involved in the suppression of p53 expression. It has also been reported that p53 and p63 proteins suppress the transcription of heat shock protein-encoding genes via their promoter binding sites for the transcription factor NF-Y40. The suppression of heat shock protein expression by kojic acid may play an important role in its antitumor activity. The 70$\mathrm{kDa}$ heat shock cognate protein (HSP7C) is a member of the HSP70 heat shock protein family. HSC/HSP70 function is regulated by the chaperone Bcl-2-associated gene product-1 (BAG-1), which acts as a coupling factor between HSC/HSP70 and a proteasome component ${ }^{41}$. BAG-family proteins also contain the BAG-domain, which mediates a direct 
interaction with HSP70, the ATPase domain and a number of client proteins, including the protein kinase Raf-142. Raf-1 is an important signaling molecule that functions in the Ras pathway to transmit mitogenic, differentiative and oncogenic signals to the downstream kinases MEK and ERK ${ }^{43}$. According to the BioCarta pathway of melanogenesis, Raf-1, Ras, MEK and ERK are important regulatory proteins in melanocytes. HSP70 also has an important role in the cotranslational maturation pathway of the human type I membrane glycoprotein tyrosinase, which interacts with calreticulin and BiP/Grp78 during its ER transit ${ }^{44}$. Thus, the HSP7C associated with heat shock proteins can interact with regulatory molecules in the melanogenesis pathway, in which these proteins are upstream regulators of other melanogenic enzymes, including tyrosinase and tyrosinase related protein-1.

The protein phosphatase 2AAA (or PP2A) was found to interact with hyperphosphorylated/desensitized Raf-145. 2AAA is required for recycling of Raf-1 to an activation-competent state and for melanosome aggregation, and it interacts with p53, Raf-1 and Bcl-2. 2AAA is also a major Ser/Thr phosphatase implicated in the regulation of many cellular processes, including many signal transduction pathways and cell-cycle progression ${ }^{46}$. In cancer cells, 2AAA has been proposed to negatively regulate cellular growth and might function as a tumor suppressor ${ }^{47}$. Confirmation by Western blot analysis showed 2AAA is upregulated in kojic acid-treated A375 melanoma cells at 48 and $72 \mathrm{~h}$. It has been suggested that kojic acid may stimulate the expression of 2AAA, leading to suppression of signaling molecules in melanogenesis. According to 2-D gel analysis, 6 isoforms of vimentin were downregulated in kojic acid-treated A375 melanoma cells. It is well known that vimentin regulates cell migration, membrane trafficking, granular secretion, protein kinase activation and regulation of stress response proteins ${ }^{48}$. The modification of vimentin isoforms might be caused by phosphorylation, which has a central role in regulating the dynamics of vimentin assembly into polymers as well as in regulating the connections between intermediate filament (IF) and IF-associated proteins ${ }^{49}$. There are many phosphorylation events affecting vimentin. For example, phosphorylation by Raf-1associated vimentin kinase results in the proper structure of vimentin filaments ${ }^{50}$ and phosphorylation by protein kinase $\mathrm{C}$ (PKC) creates isoforms that participate in the regulation of organelle movement in melanophores and many signal transduction pathways involving tyrosine kinase, nucleotide exchange factor, serine/threonine kinase, Raf- 1 , PKC, cytoskeletal protein actin, proteosome component and HSC7051. In addition to phosphorylation, the different spots of vimentin seen on the 2-D gel may be due to association with other molecules, such as protein kinase $C$, integrin or other associated proteins in signal transduction pathways. Changes in vimentin expression levels are also generated by stress, such as heat shock and oxidative stress ${ }^{52}$. Cleavage of vimentin precedes the recognition of cytoskeletal filaments in apoptosis and programmed cell death mechanisms. Downregulation of vimentin expression inhibits migration and invasion of colon and breast cancer cell lines ${ }^{53}$. According to our Western blot analysis, down-regulated vimentin may suppress tumorigenesis and regulate apoptosis in kojic acid-treated A375 melanoma cells. Moreover, the presence of more than one protein band recognized by the monoclonal antibody of vimentin may be caused by post-translational modifications. Another protein that leads to tumor suppression is pyruvate kinase isoenzyme (KPYM). It is a key sensor for energy consumption and regulation of the glycolytic pathway, in which KPYM links energy-rich metabolites from the flow of glucose carbons to nucleic acid ${ }^{4}$. In yeast cells, overexpression of pyruvate kinases leads to an inhibition of cell proliferation by depletion of glycolytic phosphometabolites. Likewise, pyruvate kinases in eukaryotic cells 
are involved in such fundamental processes as cell proliferation, tumor formation and apoptosis 55 . Energy used by cancer cells is preferentially produced from glycolysis, in which p53 was found to modulate the balance between the utilization of the respiratory and glycolytic pathways ${ }^{56}$. According to our results from 2-D PAGE and Western blot data, KPYM upregulated in kojic acid-treated A375 melanoma cells may play an important role in the suppression of tumor growth and metastasis. In summary, we identified a number of differentially expressed proteins in kojic acid-treated A375 melanoma cells. Most of these proteins were key factors involved in a wide variety of cellular processes, including cell proliferation, regulation of gene expression, signaling, and chromatin and cytoskeleton organization. Interestingly, our proposed interaction network reveals proteins associated with the regulation of apoptosis, which may lead to suppression of the melanogenesis and tumorigenesis of cancer cells. Moreover, these proteins may be useful biomarkers for use in diagnostic and therapeutic applications of skin cancer. Further functional studies of these proteins may lead to better understanding of the pathogenic mechanisms and cellular response to kojic acid treatment.

According to our investigation of the toxicogenomic effects of arbutin on the gene expression profile of A375 melanoma cells using microarray ${ }^{26}$, there was no growth inhibition or morphological change of A375 melanoma cells after $72 \mathrm{~h}$ in the presence of 8 $\mu \mathrm{g} / \mathrm{ml}$ arbutin, which is a mild arbutin concentration and safe for human skin. The gene expression data showed some tumor suppressor genes as biomarkers in A375 melanoma cells. Although one gene makes one protein, post-translational modifications of proteins can lead to changes in biological and physiological functions that may not result from gene modification. To examine the effects of arbutin on protein expression levels in A375 melanoma cells, the same concentration of arbutin $(8 \mu \mathrm{g} / \mathrm{ml})$ at different treatment times of 24, 48 and $72 \mathrm{~h}$ was used. Among the differentially expressed proteins validated by RTqPCR, VIME, 14-3-3G and VDAC-1 were found to interact with Raf-1. VIME, an IF protein, is the major cytoskeleton component of developing cancer cells. VIME is phosphorylated by Raf-1-associated vimentin kinase, resulting in the regulation of the vimentin filament structure $^{57}$. VIME is also a target for phosphorylation by PKC, which is involved in many signal transduction pathways including the regulation of organelle movement in melanophores 58 , cytoskeletal function and programmed cell death (apoptosis) ${ }^{59}$. In carcinoma cell lines, downregulation of VIME expression resulted in impaired migration and adhesion ${ }^{60}$. Likewise, our RT-qPCR results show that VIME is downregulated in arbutin-treated A375 melanoma cells, suggesting an effect on the IF network that ultimately leads to impaired migration and adhesion of A375 melanoma cells. Because VIME is involved in the regulation of stress response proteins ${ }^{61}$, the heat shock protein ENPL, also found to be downregulated in A375 melanoma cells in this study, may be involved in VIME regulation. ENPL is a member of the heat shock protein HSP90 complex found in the melanosome. It is well known that heat shock proteins are overexpressed in a wide range of human cancers and are implicated in tumor cell proliferation, differentiation and recognition by the immune system ${ }^{62}$. Therefore, the ENPL downregulated in arbutin-treated A375 melanoma cells may suppress tumor progression and metastasis and cause the decreased immune response.

ENPL was also found to be associated with other types of heat shock proteins and interacts with p53 and 14-3-3 proteins, both of which play important roles in apoptosis. 14-3-3 proteins act as control points for many cellular processes and therefore play significant roles in cell-fate determination and in several apoptotic pathways in animals ${ }^{63}$. 14-3-3 proteins 
have been reported to have important interactions with other regulatory proteins. They display important anti-apoptotic characteristics by inhibiting the pro-apoptotic Bcl-2antagonist of cell death (BAD) and the transcription factor FKHRL-1. Major roles ascribed to the mammalian 14-3-3 proteins include activation of tyrosine and tryptophan hydroxylases, regulation of $\mathrm{PKC}$, exocytosis, especially in mediating interactions between protein kinases, and other signal transduction proteins ${ }^{64}$. 14-3-3 was found to activate the Ras-Raf mitogenic pathway and can elicit a physiologically significant activation of Raf- 1 in mammalian cells 65 . Because 14-3-3 proteins are involved in a great number of interactions, the effects of knocking out or overexpressing specific 14-3-3 genes or 14-3-3 target genes will likely have effects on other 14-3-3-regulated cellular processes. Studies have found that 14-3-3 proteins activate p53 function in vivo; thus, the up-regulation of 14-3-3G protein expression in arbutin-treated A375 melanoma cells implies that arbutin can stimulate changes in signal transduction pathways by stabilizing or increasing the expression of 14-3-366. The increase in protein expression might involve a critical response of cell proliferation, differentiation and apoptosis of arbutin-treated A375 melanoma cells. The change in the expression of 14-3-3 might alter the biological activities of p53, RAF-1, CLIC1, ENPL, Bcl2 and kinesin-like proteins. Interestingly, the partial functions of ENPL and VDAC-1 on ion channel activity and ion sequestering ability are involved in energy metabolism, which is important for survival. The cellular location of these proteins at the cell membrane and mitochondria implies that the effect of arbutin on membrane and mitochondria may cause changes in cellular physiology and metabolic events. VDAC-1, a major mitochondrial outer membrane transporter, is a component of the permeability transition (PT) and plays an important role in apoptosis by participating in the release of intermembrane space proteins, including cytochrome $\mathrm{c}$, and by its involvement in $\mathrm{Ca}^{2+}$ signaling ${ }^{67}$. VDAC- 1 has also been found to be associated with the Bax/Bak and Bcl-2 families of proteins, which are essential regulators of cell death and exert their primary pro- or anti-apoptotic roles at the mitochondrial outer membrane. Therefore, the upregulation of VDAC- 1 expression in arbutin-treated A375 melanoma cells may play a crucial role in mitochondria-mediated apoptosis, mitochondrial membrane permeability transition and intracellular $\mathrm{Ca}^{2+}$ transport. Alternatively, p53 tumor suppressor proteins have been reported to have additional roles in the regulation of glycolysis.

In this study, four proteins identified as glycolytic enzymes, G3P, ENOA, G6PD and MDHM, were found to be involved in glucose metabolism, but only ENOA was validated by RT-PCR. The low expression of ENOA in arbutin-treated A375 melanoma cells may be implicated in metastasis and may also change the associated signaling pathways that modulate cellular metabolism. In addition to regulating glycolysis in cancer cells, p53 also helps to regulate both apoptosis and intracellular reactive oxygen species (ROS) levels. Additionally, peroxiredoxin-1 (PROX1) is one of the antioxidant enzymes and is involved in cellular proliferation and differentiation. The ability of PROX1 to enhance cell survival is traditionally attributed to its capacity to remove ROS68. Indeed, lower PROX1 activity increased levels of ROS and induced p53 expression. Therefore, the level of p53 is a major determinant of the effect of decreased PROX1 expression on tumor growth and the response of cells to treatment. This agrees with our results showing up-regulated p53 and downregulated PROX1 in arbutin-treated A375 melanoma cells. In addition to energy metabolism, arbutin may also affect nucleotide metabolism because ribonucleoproteins (HNRH1, ROA2, ROA1), ribonuclease inhibitor (RINI) and inosine-5'-monophosphate dehydrogenase (IMDH2) were affected by arbutin treatment. IMDH2 is a regulatory enzyme of guanine 
nucleotide biosynthesis and is also strategically positioned in the metabolic pathway of thiopurines. Increased mRNA expression levels and enzymatic activity of IMPDH2 have been observed in rapidly proliferating tumor cells. IMDH2 has been established as an anticancer target. Therefore, downregulation of IMDH2 may suppress nucleotide biosynthesis, cell proliferation and malignancy of A375 melanoma cells. According to our proteomic results, many identified proteins, including heat shock proteins, glucoseregulated proteins and other proteins, were found to be associated with p53, which is a major tumor-suppressor protein and plays significant roles in many biological processes in cancer development. Moreover, p53 has biological roles in pigmentation. Tumor suppressor p53 could down- and upregulate tissue-specific expression of the tyrosinase gene in human melanoma cell lines. Therefore, arbutin has potent effects on both protein and gene expression and leads to the suppression of melanogenesis and tumorigenesis of A375 melanoma cells. However, the correlation of protein and gene expression with biological function will be further studied to better understand the effect of arbutin on the biology of A375 malignant melanoma cancer cells. In summary, we identified differentially expressed proteins in arbutin-treated A375 melanoma cells. Most of these proteins were key players in a wide variety of cellular processes, including cell proliferation, regulation of protein expression and signaling pathways.

\section{References}

[1] Braud, F.; Khayat, D.; Kroon, B. B. R.; Valdagni, R.; Bruzzi, P. \& Cascinelli, N. (2003). Malignant melanoma. Critical Reviews in Oncology/Hematology, 47(1), 35-63.

[2] Cheng, S. L.; Huang-Liu, R.; Sheu, J. N.; Chen, S. T.; Sinchaikul, S. \& Tsay G. J. (2007). Toxicogenomics of A375 human malignant melanoma cells. Pharmacogenomics, 8 (8), 1017-1036.

[3] Soengas, M. S.; Capodieci, P.; Polsky, D.; Mora, J.; Esteller, M.; Opitz-Araya, X.; McCombie, R.; Herman, J. G.; Gerald, W. L.; Lazebnik, Y. A.; Cordón-Cardó, C. \& Lowe, S. W. (2001). Inactivation of the apoptosis effector Apaf-1 in malignant melanoma. Nature, 409, 207-211.

[4] Pennie, W. D.; Tugwood, J. D.; Oliver, G. J. A. \& Kimber, I. (2000). The principles and practical of toxicogenomics: applications and opportunity. Toxicol. Sci., 54 (2), 277283.

[5] Kwak, M. Y. \& Rhee, J. S. (1992). Cultivation characteristics of immobilized Aspergillus oryzae for kojic acid production. Biotechnol Bioeng, 39 (9), 903-906.

[6] Masse, M. O.; Duvallet, V.; Borremans, M. \& Goeyens, L. (2001). Identification and quantitative analysis of kojic acid and arbutin in skin-whitening cosmetics. International Journal of Cosmetic Science, 23(4), 219-232.

[7] Beard, R. L. \& Walton, G. S. (1969). Kojic acid as an insecticidal mycotoxin. Journal of Insect Physiology, 14(1), 53-59.

[8] Higa, Y.; Ohkubo, A.; Kitajima, S.; Moriyasu, M. \& Kariya K. (2002). Effects of kojic acid on thyroidal functions in rats by single-dose administration and in cultured rat thyroid cells (frtl-5 cells). The Journal of Toxicological Sciences, 27, 423-431.

[9] Lee, H. F.; Boltjes, B. \& Eisenman, W. (1950). Kojic acid as an inhibitor of tubercle bacilli. Am. Rev. Tuberculosis, 61(5), 738-741.

[10] Nohynek, G. J.; Kirkland, D.; Marzin, D.; Toutain, H.; Leclerc-Ribaud, C. \& Jinnai, H. (2004). An assessment of the genotoxicity and human health risk of topical use of 
kojic acid [5-hydroxy-2-(hydroxymethyl)-4H-pyran-4-one]. Food and Chemical Toxicology, 42(1), 93-105.

[11] Hirose, M.; Nishikawa, A.; Shibutani, M. \& Mitsumori K. (2001). Environmental agents, endocrine disrupting chemicals and rat thyroid carcinogenesis. Joural of Toxicologic Pathology, 14, 71-77.

[12] Takizawa, T.; Imai, T.; Onose, J. I.; Ueda, M.; Tamura, T.; Mitsumori, K.; Izumi, K. \& Hirose M. (2004). Enhancement of hepatocarcinogenesis by kojic acid in rat twostage models after initiation with $\mathrm{N}$-bis(2-hydroxypropyl)nitrosamine or $\mathrm{N}$ diethylnitrosamine. Toxicology Sciences, 81, 43-49 (2004).

[13] Tomita, K.; Fukuda, M. \& Kawasaki, K. (1990). Mechanism of arbutin inhibitory effect on melanogenesis and effect on the human skin with cosmetic use. Fragrance J. 6, 72-77.

[14] Sugimoto, K.; Nishimura, T.; Nomura, K.; Sugimoto, K. \& Kuriki, T. (2004). Inhibitory effects of a-arbutin on melanin synthesis in cultured human melanoma cells and a threedimensional human skin model. Biol. Pharm. Bull., 27(4), 510-514.

[15] Slominski, A.; Tobin, D. J.; Shibahara, S. \& Wortsman, J. (2004). Melanin pigmentation in mammalian skin and its hormonal regulation. Physiol. Rev., 84, 1155-1228.

[16] Shibata, T.; Prota, G. \& Mishima, Y. (1993). Non-melanosomal regulatory factors in melanogenesis. J. Invest. Dermatol., 100, S274-S280.

[17] Mantripragada, K. K.; Buckley, P. G.; Stahl, T. D. \& Dumanski J. P. (2004). Genomic microarrays in the spotlight. Trends in Genetics, 20(2), 87-94.

[18] Cheng, S. L.; Liu, H. R.; Sheu, J. N.; Chen, S. T.; Sinchaikul, S. \& Tsay, G. J. (2006). Toxicogenomics of kojic acid on gene expression profiling of A375 human malignant melanoma cells. Biol. Pharm. Bull., 29, 655-669.

[19] O'Donnell, R.; Holland, J. W.; Deeth, H. C. \& Alewood, P. (2004). Milk proteomics. Int. Dairy J., 14, 1013-1023.

[20] Larsen, L. B.; Wedholm, A.; Møller, H. S.; Andrén, A. \& Lindmark-Månsson, H. (2007). Proteomic study of regressions between milk yield and whey protein composition. J. Anim. Feed Sci., 16, 200-206.

[21] Chevalier, F. \& Kelly, A. L. (2010). Proteomic quantification of disulfide-linked polymers in raw and heated bovine milk. J. Agric. Food Chem., 58(12), 7437-7444.

[22] Nelson, D. L. \& Cox, M. M. (2005). Amino acids, Peptides, and Proteins. New York: W. H. Freeman and Company. pp. 79-95.

[23] Miralles, B.; Leaver, J.; Ramos, M. \& Amigo, L. (2003). Mass mapping analysis as a tool for the identification of genetic variants of bovine $\beta$-casein. Journal of Chromatography A, 1007(1-2), 47-53.

[24] Burdock, G. A.; Soni, M. G. \& Carabin, I. G. (2001). Evaluation of health aspects of kojic acid in food. Toxicol. Pharmacol., 33, 80-101.

[25] Fujino, T.; Navaratnam, N. \& Scott, J. (1998). Human apolipoprotein B RNA editing deaminase gene (APOBEC1). Genomics, 47, 266-275.

[26] Cheng, S. L.; Liu, R. H.; Sheu, J. N.; Chen, S. T.; Sinchaikul, S. \& Tsay, G. J. (2007). Toxicogenomics of A375 human malignant melanoma cells treated with arbutin. J. Biomed. Sci., 14, 87-105.

[27] Tiedtke, J.; Morel, J. \& Marks, O. (2004). Depigmentation factor bioflavonoids-a safe and effective skin lightener based on encapsulated citrus bioflavonoids. Cosmetochem 2, 12-17. 
[28] Nawarak, J.; Huang-Liu, R.; Kao, S. H.; Liao, H. H.; Sinchaikul, S.; Chen, S. T. \& Cheng, S. L. (2008). Proteomics analysis of kojic acid treated A375 human malignant melanoma cells. J Proteome Res., 7: 3737-3746.

[29] Nawarak, J.; Huang-Liu, R.; Kao, S. H.; Liao, H. H.; Sinchaikul, S.; Chen, S. T. \& Cheng, S. L. (2009). Proteomics analysis of A375 human malignant melanoma cells in response to arbutin treatment. Biochim Biophys Acta., 1794: 159-167.

[30] Wiechers, J. W.; Rawlings, A. V.; Garcial, C.; Chesne, C.; Balaguer, P.; Nicolas, J. C.; Corre, S. \& Galibert, M. D. (2005). A new mechanism of action for skin whitening agents: binding to the peroxisome proliferator-activated receptor. Int. J. Cosmetic Sci., 27(2), 123-132.

[31] Kunz, M.; Ibrahim, S. M.; Koczan, D.; Scheid, S.; Thiesen, H. J. \& Gross, G. (2004). DNA microarray technology and its applications in dermatology. Exp. Dermatol., 13(10), 593-606.

[32] Petersen-Mahrt, S. K. \& Neuberger, M. S. (2003). In vitro deamination of cytosine to uracil in single-stranded DNA by apolipoprotein B editing complex catalytic subunit 1 (APOBEC1). J. Biol. Chem., 278, 19583-19586.

[33] Morgan, H. D.; Dean, W.; Coker, H. A.; Reik, W. \& Petersen-Mahrt, S. K. (2004). Activation-induced cytidine deaminase deaminates 5-methylcytosine in DNA and is expressed in pluripotent tissues. J. Biol. Chem., 279, 52353-52360.

[34] Beale, R. C. L.; Petersen-Mahrt, S. K.; Wat, I. N.; Harris, R. S.; Rada, C. \& Neuberger, M. S. (2004). Comparison of the differential context-dependence of DNA deamination by APOBEC enzymes: correlation with mutation spectra in vivo. J. Mol. Biol., 337(3), 585-596.

[35] Chester, A.; Scott, J.; Anant, S. \& Navaratnam, N. (2000). RNA editing: cytidine to uridine conversion in apolipoprotein B mRNA. Biochim. Biophys. Acta, 1494(1-2), 113.

[36] Tiedtke, J.; Morel, J. \& Marks, O. (2004). Depigmentation factor bioflavonoids-a safe and effective skin lightener based on encapsulated citrus bioflavonoids. Cosmetochem, 2, 12-17.

[37] Beere, H. M. (2004). The stress of dying: the role of heat shock proteins in the regulation of apoptosis. J. Cell Sci., 117, 2641-2651.

[38] Ciocca, D. R. \& Calderwood, S. K. (2005). Heat shock proteins in cancer: diagnostic, prognostic, predictive, and treatment implications. Cell Stress Chaperones, 10, 86-103.

[39] Muller, L.; Schaupp, A.; Walerych, D.; Wegele, H. \& Buchner, J. (2004). Hsp90 regulates the activity of wild type p53 under physiological and elevated temperatures. J. Biol. Chem. 279, 48846-48854.

[40] Wu, G.; Osada, M.; Guo, Z.; Fomenkov, A.; Begum, S.; Zhao, M.; Upadhyay, S.; Xing, M.; Wu, F.; Moon, C.; Westra, W. H.; Koch, W. M.; Mantovani, R.; Califano, J. A.; Ratovitski, E.; Sidransky, D. \& Trink, B. (2005). $\Delta$ Np63alpha up-regulates the Hsp70 gene in human cancer. Cancer Research, 65, 758-766.

[41] Luders, J.; Demand, J. \& Hohfeld, J. (2000). The ubiquitin-regulated BAG-1 provides a link between the molecular chaperones $\mathrm{Hsc}_{70} / \mathrm{Hsp}_{70}$ and the proteosome. J. Biol. Chem. 275, 4613-4617.

[42] Takayama, S.; Bimston, D. N.; Matsuzawa, S.; Freeman, B. C.; Aime-Sempe, C.; Xie, Z.; Morimoto, R. I. \& Reed, J. C. (1997). BAG-1 modulates the chaperone activity of Hsp70/Hsc70. EMBO J., 16, 4887-4896. 
[43] Wang, N.; Daniels, R. \& Hebert, D. N. (2005). The cotranslational maturation of the type I membrane glycoprotein tyrosinase: The heat shock protein 70 system hands off to the lectin-based chaperone system. Mol. Biol. Cell 16, 3740-3752.

[44] Popescu, C. I.; Paduraru, C.; Dwek, R. A. \& Petrescu, S. M. (2005). Soluble tyrosinase is an endoplasmic reticulum (ER)-associated degradation substrate retained in the ER by calreticulin and BiP/GRP 78 and not calnexin. J. Biol. Chem., 280, 13833-13840.

[45] Dougherty, M. K.; Muller, J.; Ritt, D. A.; Zhou, M.; Zhou, X. Z.; Copeland, T. D.; Conrads, T. P.; Veenstra, T. D.; Lu, K. P. \& Morrison, D. K. (2005). Regulation of Raf-1 by direct feedback phosphorylation. Mol. Cell, 17(2), 215-224.

[46] Janssens, V. \& Goris, J. (2001). Protein phosphatase 2A: a highly regulated family of serine/threonine phosphatases implicated in cell growth and signalling. Biochem. J., $353,417-439$.

[47] Schonthal, A. H. (2001). Role of serine/threonine protein phosphatase 2A in cancer. Cancer Letter, 170, 1-13.

[48] Wang, N. \& Stamenovic, D. (2002). Mechanics of vimentin intermediate filaments. J. Muscle Res. Cell Motil., 23, 535-540.

[49] Ku, N. O.; Liao, J. \& Omary, M. B. (1998). Phosphorylation of human keratin 18 serine 33 regulates binding to 14-3-3 proteins. EMBO J., 17, 1892-1906.

[50] Janosch, P.; Kieser, A.; Eulitz, M.; Lovric, J.; Sauer, G.; Reichert, M.; Gounari, F.; Büscher, D.; Baccarini, M.; Mischak, H. \& Kolch, W. (2000). The Raf-1 kinase associates with vimentin kinases and regulates the structure of vimentin filaments. FASEB J., 14, 2008-2021.

[51] Ivaska, J.; Vuoriluoto, K.; Huovinen, T.; Izawa, I.; Inagaki, M. \& Parker, P. J. (2005). PKC $\varepsilon$-mediated phosphorylation of vimentin controls integrin recycling and motility. EMBO J., 24, 3834-3845.

[52] Paron, I.; D’Elia, A.; D'Ambrosio, C.; Scaloni, A.; D'Aurizio, F.; Prescott, A.; Damante, G. \& Tell, G. (2004). A proteomic approach to identify early molecular targets of oxidative stress in human epithelial lens cells. Biochem. J., 378, 929-937.

[53] Sheikh, M. S. \& Fornace Jr, A. J. (2000). Death and decoy receptors and p53-mediated apoptosis. Leukemia ,14, 1509-1513.

[54] Ginisty, H.; Sicard, H.; Roger, B. \& Bouvet, P. (1999). Structure and functions of nucleolin. J. Cell Sci., 112, 761-772.

[55] Daniely, Y.; Dimitrova, D. D. \& Borowiec, J. A. (2002). Stress-dependent nucleolin mobilization mediated by p53-nucleolin complex formation. Mol. Cell. Biol., 22, 6014-6022.

[56] Brazill, D. T.; Thormer, J. \& Martin, S. (1997). Mck1, a member of the glycogen synthase kinase 3 family of protein kinase, is a negative regulator of pyruvate kinase in the yeast Saccharomyces cerevisiae. J. Bacteriol., 179, 4415-4418.

[57] Janosch, P.; Kieser, A.; Eulitz, M.; Lovric, J.; Sauer, G.; Reichert, M.; Gounari, F.; Büscher, D.; Baccarini, M.; Mischak, H. \& Kolch, W. (2000).The Raf- ${ }_{-1}$ kinase associates with vimentin kinases and regulates the structure of vimentin filaments. FASEB J., 14, 2008-2021.

[58] Ivaska, J.; Vuoriluoto, K.; Huovinen, T.; Izawa, I.; Inagaki, M. \& Parker, P. J. (2005). PKCE-mediated phosphorylation of vimentin controls integrin recycling and motility. EMBO J., 24, 3834-3845. 
[59] Yang, X.; Wang, J.; Liu, C.; Grizzle, W. E.; Yu, S.; Zhang, S.; Barnes, S.; Koopman, W. J.; Mountz, J. D.; Kimberly, R. P. \& Zhang, H. G. (2005). Cleavage of p53-vimentin complex enhances tumor necrosis factor-related apoptosis-inducing ligandmediated apoptosis of rheumatoid arthritis synovial fibroblasts. Am. J. Pathol., 167(3), 705-719.

[60] McInroy, L. \& Maatta, A. (2007). Down-regulation of vimentin expression inhibits carcinoma cell migration and adhesion. Biochem. Biophys. Res. Comm., 360, 109-114.

[61] Benitez-King, G. (2000). PKC activation by melatonin modulates vimentin intermediate filament organization in N1E-115 cells. J. Pineal. Res., 29(1), 8-14.

[62] Ciocca, D. R. \& Calderwood, S. K. (2005). Heat shock proteins in cancer: diagnostic, prognostic, predictive, and treatment implications. Cell Stress Chaperones, 10(2), 86103.

[63] Tzivion, G. \& Avruch, J. (2002). 14-3-3 proteins: active cofactors in cellular regulation by serine/threonine phosphorylation. J. Biol. Chem., 277, 3061-3064.

[64] Aitken, A. (1996). 14-3-3 and its possible role in coordinating multiple signaling pathways. Trends Cell Biol., 6(9), 341-347.

[65] Li, S.; Janosch, P.; Tanji, M.; Rosenfeld, G. C.; Waymire, J. C.; Mischak, H.; Kolch, W. \& Sedivy, J. M. (1995). Regulation of Raf-1 kinase activity by the 14-3-3 family of proteins. EMBO J., 14(4), 685-696.

[66] Waterman, J. L.; Shenk, J. L. \& Halozonetis, T. D. (1995).The dihedral symmetry of the p53 tetramerization domain mandates a conformational switch upon DNA binding. EMBO J. 14(3), 512-519.

[67] Shoshan-Barmatz, V. \& Gincel, D. (2003). The voltage-dependent anion channel: characterization, modulation, and role in mitochondrial function in cell life and death. Cell Biochem. Biophys., 39, 279-292.

[68] Kim, Y. J.; Ahn, J. Y.; Liang, P.; Ip, C.; Zhang, Y. \& Park, Y. M. (2007). Human prx1 gene is a target of Nrf2 and is up-regulated by hypoxia/reoxygenation: implication to tumor biology. Cancer Res., 67, 546-554. 


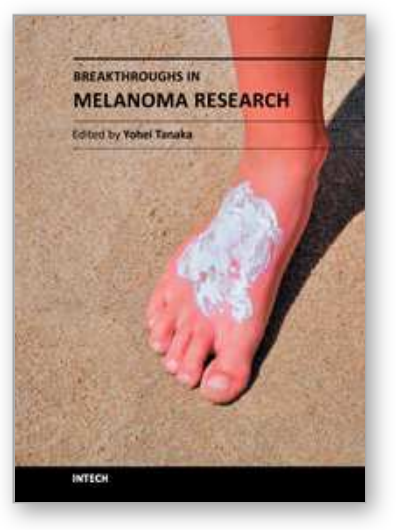

\author{
Breakthroughs in Melanoma Research \\ Edited by Dr Yohei Tanaka
}

ISBN 978-953-307-291-3

Hard cover, 628 pages

Publisher InTech

Published online 30, June, 2011

Published in print edition June, 2011

Melanoma is considered to be one of the most aggressive forms of skin neoplasms. Despite aggressive researches towards finding treatments, no effective therapy exists to inhibit the metastatic spread of malignant melanoma. The 5-year survival rate of metastatic melanoma is still significantly low, and there has been an earnest need to develop more effective therapies with greater anti-melanoma activity. Through the accomplishment of over 100 distinguished and respected researchers from 19 different countries, this book covers a wide range of aspects from various standpoints and issues related to melanoma. These include the biology of melanoma, pigmentations, pathways, receptors and diagnosis, and the latest treatments and therapies to make potential new therapies. Not only will this be beneficial for readers, but it will also contribute to scientists making further breakthroughs in melanoma research.

\title{
How to reference
}

In order to correctly reference this scholarly work, feel free to copy and paste the following:

Jung-Feng Hsieh, Shui-Tein Chen and Sun-Long Cheng (2011). Molecular Profiling of A375 Human Malignant Melanoma Cells Treated with Kojic Acid and Arbutin, Breakthroughs in Melanoma Research, Dr Yohei Tanaka (Ed.), ISBN: 978-953-307-291-3, InTech, Available from: http://www.intechopen.com/books/breakthroughs-inmelanoma-research/molecular-profiling-of-a375-human-malignant-melanoma-cells-treated-with-kojic-acidand-arbutin

\section{INTECH}

open science | open minds

\author{
InTech Europe \\ University Campus STeP Ri \\ Slavka Krautzeka 83/A \\ 51000 Rijeka, Croatia \\ Phone: +385 (51) 770447 \\ Fax: +385 (51) 686166 \\ www.intechopen.com
}

\author{
InTech China \\ Unit 405, Office Block, Hotel Equatorial Shanghai \\ No.65, Yan An Road (West), Shanghai, 200040, China \\ 中国上海市延安西路65号上海国际贵都大饭店办公楼 405 单元 \\ Phone: +86-21-62489820 \\ Fax: +86-21-62489821
}


(C) 2011 The Author(s). Licensee IntechOpen. This is an open access article distributed under the terms of the Creative Commons Attribution 3.0 License, which permits unrestricted use, distribution, and reproduction in any medium, provided the original work is properly cited. 